Hardy-Ramanujan Journal

Vol.17 (1994) 1-31

\title{
ESTIMATE OF SUMS OF DIRICHLET SERIES
}

\section{Shituo Lou and Qi Yao}

\section{§ 1. INTRODUCTION.}

Let $x$ be a large positive number, $\varepsilon$ be a small positive number and $k$ be a finite integer. Let $y=x^{\theta}, 1 / 2<\theta<7 / 12, T=x^{1-\theta+\varepsilon / 2}$, and let $M_{1}, \cdots, M_{k}$ and $L$ be real numbers such that

$$
M_{1} \cdots M_{k} L=x / 2
$$

$U_{1}, \cdots, U_{k}$ and $W$ be real numbers such that

$$
W \ll L^{1 / 2-\varepsilon}
$$

and

$$
U_{i} \ll M_{i}^{1 / 2}, 1 \leq i \leq k .
$$

Let $A$ be a fixed integer, and $|S|$ be the number of elements of set $S$. We discuss the set $S\left(U_{1}, \cdots, U_{k}, W\right)$ which satisfies following conditions, for $1 \leq i \leq k$ and $j$ be a positive integer,

(1.4) $\left|S\left(U_{1}, \cdots, U_{k}, W\right)\right| \ll U_{i}^{-2 j}\left(M_{i}^{j}+T\right)(\log x)^{A}$ and $\left|S\left(U_{1}, \cdots, U_{k}, W\right)\right| \ll$ $W^{-2 j}\left(L^{j}+T\right)(\log x)^{A}$

(1.5) $\left|S\left(U_{1}, \cdots, U_{k}, W\right)\right| \ll\left(U_{i}^{-2 j} M_{i}^{j}+U_{i}^{-6 j} M_{i}^{j} T\right)(\log x)^{A}$;

(1.6) $\left|S\left(U_{1}, \cdots, U_{k}, W\right)\right| \ll\left(W^{-2 j} L^{j}+W^{-6 j} L^{j} T\right)(\log x)^{A}$; 
(1.7) $W^{4}\left|S\left(U_{1}, \cdots, U_{k}, W\right)\right| \ll T(\log x)^{A}$.

Let $a_{j}=\log M_{j} / \log x$. In $\S 2$ we shall give some functions $h_{i}\left(a_{1}, \cdots, a_{k}\right)$ for $1 \leq i \leq 3$, such that

$$
U_{1} \cdots U_{k} W\left|S\left(U_{1}, \cdots, U_{k}, W\right)\right| \ll x^{1 / 2-\varepsilon}+x^{h_{i}\left(a_{1}, \cdots, a_{k}\right)} .
$$

In $\S 3$, we discuss those $S\left(U_{1}, \cdots, U_{k}, W\right)$ 's which satisfy one more inequality : for $1 \leq j \leq k$,

$$
\left|S\left(U_{1}, \cdots, U_{k}, W\right)\right| \ll W^{-4} U_{i}^{-2}\left(M_{j}^{2} T^{1 / 2}+M_{j}^{5 / 4} T^{3 / 4}+T\right) T^{\varepsilon} .
$$

If $T^{1 / 5} \leq M_{j} \leq T^{1 / 3}$, we replace (1.9) by

$$
\left|S\left(U_{1}, \cdots, U_{k}, W\right)\right| \ll W^{-4} U_{i}^{-2} M_{j}^{5 / 4} T^{3 / 4+\varepsilon},
$$

and, if $M_{j} \leq T^{1 / 5}$, we replace (1.9) by

$$
\left|S\left(U_{1}, \cdots, U_{k}, W\right)\right| \ll W^{-4} U_{i}^{-2} T^{1+\varepsilon} .
$$

In $\S 3$ we will give $h_{i}\left(a_{1}, \cdots, a_{k}\right)$ for $4 \leq i \leq 10$ with (1.8).

Heath-Brown and Iwaniec [1] discussed the gaps between consecutive primes using sieve method. The remainder term

$$
R\left(x ; M_{1}, \cdots, M_{k}\right)=\sum_{\substack{M_{i}<m_{i} \leq 2 M_{i} \\ 1 \leq i \leq k}} a_{m_{1}, 1}, \cdots, a_{m_{k}, k} r_{m_{1} \cdots m_{k}},
$$

where

$$
r_{d}=\left[\frac{r}{d}\right]-\left[\frac{x-y}{d}\right],
$$

$M_{i}<y$ and $\left|a_{m_{i}}, i\right| \leq 1$, be considered. Applying the method which is very close to Heath-Brown and Iwaniec's, in $\S 5$, we will show that

$$
R\left(x ; M_{1}, \cdots, M_{k}\right) \ll x^{\theta-\varepsilon},
$$

if (1.8) holds with

$$
h_{i}\left(a_{1}, \cdots, a_{k}\right)<1 / 2 \text {, }
$$


In fact, they proved that (see [1]) if $\theta=11 / 20+\varepsilon, k=2, M_{1} \ll x^{0.46-\varepsilon}$, and $M_{2} \ll x^{0.48-\varepsilon},(1.13)$ holds. Consequently, they obtained that for $y=$ $x^{\theta}, \theta \geq 11 / 20+\varepsilon$,

$$
\pi(x)-x(x-y)>\frac{1}{212} \frac{y}{\log x},
$$

where $\pi(x)$ be the number of primes $\leq x$.

In [2], Heath-Brown discussed some kind of products of Dirichlet series

$$
W(s)=X(s) \prod_{j=1}^{k_{0}} Y_{j}(s)
$$

where

$$
\begin{gathered}
X(s)=\sum_{L_{0}<n \leq 2 L_{0}} n^{-s}, \\
Y_{j}(s)=\sum_{M_{j}<n \leq 2 M_{j}} b_{m} n^{-s},\left|b_{m}\right| \leq 1 ;
\end{gathered}
$$

and

$$
L_{0} \prod_{j=1}^{k_{0}} M_{j}=\frac{x}{2} .
$$

Using Heath-Brown's method that was used in [2], in $\S 5$, we shall show that

$$
\int_{T}^{2 T}\left|W\left(\frac{1}{2}+i t\right)\right| d t \ll x^{\frac{1}{2}} \exp \left(-(\log x)^{\frac{1}{3}}(\log \log x)^{\frac{2}{3}}\right)
$$

for

$$
T_{1} \leq T \leq x^{1-\theta+\varepsilon},
$$

where $\theta$ is a fixed positive constant with $1 / 2<\theta<1$, and

$$
T_{1}=\exp \left((\log x)^{\frac{1}{3}}(\log \log x)^{-\frac{1}{3}}\right),
$$

if (1.8) holds with (1.14).

In [3], and [4], we shall apply the results of this paper to investigate gaps between consecutive primes and prove that (1.11) holds for $\theta=11 / 20+\varepsilon$ 
in [3] and $\theta=6 / 11+\varepsilon$ in [4]. In Section 4, we also discuss the case of $\theta=11 / 20+\varepsilon$ that will be used in [3] to prove that

$$
0.99 \frac{y}{\log x}<\pi(x)-\pi(x-y)<1.01 \frac{y}{\log x}
$$

In Section 4 we discuss the case of $\theta=6 / 11+\varepsilon$ as well that will be used in [4] to prove that

$$
\frac{0.969 y}{\log x}<\pi(x)-x(x-y)<\frac{1.031 y}{\log x}
$$

for $\theta=6 / 11+\varepsilon$.

In this paper $\delta$ be another positive small number with $\delta \ll \varepsilon$ and may be difference in some paragraphs. Also, we use

$$
c \delta<\delta
$$

\section{§2. ESTIMATE OF $\left\{S\left(U_{1}, \cdots, U_{k}, W\right)\right\}$.}

In this section we estimate $\left|S\left(U_{1}, \cdots, U_{k}, W\right)\right|$. First we divide $M_{1}, \cdots, M_{k}$, and $L$ into $k_{0}+1$ parts (in this paper $2 \leq k_{0} \leq 6$ ), denote the products of elements in one part by $N_{1}, \cdots, N_{k_{0}}$, and $L_{0}$, respectively. Also denote the product of those $U_{h}$ 's or $W$ that correspond to $N_{j}$ or $L_{0}$ by $U_{j}$ or $W$, again. We now replace $(1.1),(1.2)$ and $(1.3)$ by

$$
\begin{gathered}
N_{1} \cdots N_{k_{0}} L_{0}=\frac{x}{2}, \\
U_{j} \leq N_{j}^{\frac{1}{2}} A_{j}, \quad 1 \leq j \leq k_{0},
\end{gathered}
$$

and

$$
W \leq L_{0}^{\frac{1}{2}} A_{0}
$$

where $A_{j}$ (or $\left.A_{0}\right)=x^{-\delta}$ if $L$ belongs to $N_{j}$ (or $L_{0}$ ), otherwise $A_{j}=1$. Thus we have that

$$
W \prod_{i=1}^{k} U_{i} \ll\left(L_{0} \prod_{i=1}^{k} M_{i}\right)^{\frac{1}{2}-\delta} \ll x^{\frac{1}{2}-\delta}
$$


It is clear that if $L_{0}$ satisfies (1.6) with $i=2$ and $L_{0} \ll T^{1 / 2}$, then $L_{0}$ satisfies (1.7). In this paper, assume that

$$
L_{0} \ll T^{1 / 2} \text { if } L_{0} \neq L .
$$

Replacing $L$ by $L_{0}$, we shall show that the function $\left|S\left(U_{1}, \cdots, U_{k 0}, W\right)\right|$ with (1.4), (1.5), (1.6), (1.7) and (2.4) such that (1.8).

We now renew the notations $a_{i}=\log N_{i} / \log x, i=1, \cdots, k_{0}, \sigma=$ $\log L_{0} / \log x=1-a_{1}-\cdots-a_{k_{0}}$ and $t_{0}=\log T / \log x$. Let

$$
F=\left|S\left(U_{1}, \cdots, U_{k 0}, W\right)\right| x^{-\delta}
$$

We replace $\left|S\left(U_{1}, \cdots, U_{k 0}, W\right)\right|$ by $F$, it makes that we can drop the factor $\log ^{A} x$ in the right hand side of (1.4), (1.5), (1.6) and (1.7), and drop $T^{\varepsilon}$ in (1.9).

If $F \geq 2 U_{i}^{-2 j} N_{i}^{j}$, then we have that

$$
N_{i} \ll T^{1 / j}
$$

since $F \leq U_{i}^{-2 j}\left(N_{i}^{j}+T\right)$.

Suppose $j$ such that

$$
\sigma \leq t_{0} / j \text { and } j=2 \text { if } \sigma \geq t_{0} / 2 .
$$

Thus, if $j>2$, we have $\sigma \leq t_{0} / j$, i.e. $W^{j} \leq T$. Then

$$
F \ll W^{-2 j} T .
$$

We prove following lemmas :

Lemma 2.1. Let $k_{0}=2$, we have

$$
U_{1} U_{2}\left|S\left(U_{1}, U_{2}, W\right)\right| \ll x^{h_{0}\left(a_{1}, a_{2}\right)+\varepsilon / 2}+x^{1 / 2-\delta},
$$

where $h_{0}\left(a_{1}, a_{2}\right)=h_{0}\left(a_{2}, a_{1}\right), a_{1}^{\prime}=\max \left\{t_{0}, a_{1}\right\}$ and

$$
h_{0}\left(a_{1}, a_{2}\right)= \begin{cases}\frac{1}{2}-\varepsilon, & a_{1} \geq a_{2} \geq t_{0} \\ \frac{t_{0}}{2}+\frac{a_{1}}{2}+\min \left\{\frac{a_{2}}{4 j}, \frac{\sigma}{6}+\frac{a_{2}}{12 j}\right\}, & \text { otherwise. }\end{cases}
$$


Proof. We have that, by (1.4), (1.5) and (1.6),

$$
\begin{gathered}
F \leq \min \left\{U_{1}^{-2}\left(N_{1}+T\right), U_{2}^{-2}\left(N_{2}+T\right), U_{1}^{-2} N_{1}+U_{1}^{-6} N_{1} T, U_{2}^{-2} N_{2}+U_{2}^{-6} N_{2} T,\right. \\
\left.W^{-2 j} L_{0}^{j}+W^{-6 j} L_{0}^{j} T, W^{-2 j} T\right\},
\end{gathered}
$$

and proceed to show that

$$
U_{1} U_{2} W F \ll x^{h_{0}\left(a_{1}, a_{2}\right)}+x^{\frac{1}{2}-\delta} .
$$

We now consider four cases :

Case 1. $F \leq 2 U_{1}^{-2} N_{1}$ and $F \leq 2 U_{2}^{-2} N_{2}$.

Choosing $k^{\prime}$ such that $L_{0}^{k^{\prime}} \geq T$, we have $F \ll W^{-2 k^{\prime}} L_{0}^{k^{\prime}}$, and then

$$
\begin{aligned}
U_{1} U_{2} W F & \ll U_{1} U_{2} W\left(U_{2}^{-2} N_{2}\right)^{\frac{1}{2}-\frac{1}{2\left(2 k^{\prime}+1\right)}}\left(U_{1}^{-2} N_{2}\right)^{\frac{1}{2}-\frac{1}{2\left(2 k^{\prime}+1\right)}}\left(W^{-2 k^{\prime}} L_{0}^{k^{\prime}}\right)^{\frac{1}{2 k^{\prime}+1}} \\
& \ll\left(U_{1} U_{2} W\right)^{\frac{1}{2 k^{\prime}+1}}\left(N_{1} N_{2} L_{0}\right)^{\frac{1}{2}-\frac{1}{2\left(2 k^{\prime}+1\right)}} \ll x^{\frac{1}{2}-\delta}
\end{aligned}
$$

by (2.4). Thus (2.12) holds. (Note : when $a_{1} \geq a_{2} \geq t_{0}$, we have that

$$
F \leq 2 U_{1}^{-2} N_{1} \text { and } F \leq 2 U_{2}^{-2} N_{2}
$$

by (1.4) with $i=1$. This completes the proof of (2.10).)

Case 2. $F \leq 2 U_{1}^{-2} N_{1}, F>2 U_{2}^{-2} N_{2}$.

In this case we have that $F \ll U_{2}^{-2} T, F \ll U_{2}^{-6} N_{2} T$ and $N_{2} \ll T$ since (1.4), (1.5) (take $\left.M_{i}=N_{i}\right)(2.7)$ and $F>2 U_{2}^{-2} N_{2}$. Thus

$$
\begin{aligned}
F \leq & 2 \min \left\{U_{1}^{-2} N_{1}, U_{2}^{-2} T, U_{2}^{-6} N_{2} T, W^{-2 i} L_{0}^{j}, W^{-2 j} T\right\} \\
+ & 2 \min \left\{U_{1}^{-2} N_{1}, U_{2}^{-2} T, U_{2}^{-6} N_{2} T, W^{-2 j} L_{0}^{j} T, W^{-2 j} T\right\} \\
\leq & 2\left(U_{1}^{-2} N_{1}\right)^{1 / 2}\left(U_{2}^{-2} T\right)^{(2 j-3) / 4 j}\left(U_{2}^{-6} N_{2} T\right)^{1 / 4 j}\left(W^{-2 j} \min \left\{L_{0}^{j}, T\right\}\right)^{1 / 2 j} \\
+ & 2 \min \left\{\left(U_{1}^{-2} N_{1}\right)^{1 / 2}\left(U_{2}^{-2} T\right)^{(2 j-3) / 4 j}\left(U_{2}^{-6} N_{2} T\right)^{1 / 4 j}\left(W^{-2 j} T\right)^{1 / 2 j},\right. \\
& \left.\left(U_{1}^{-2} N_{1}\right)^{1 / 2}\left(U_{2}^{-2} T\right)^{(2 j-1) / 4 j}\left(U_{2}^{-6} N_{2} T\right)^{1 / 12 j}\left(W^{-6 j} L_{0}^{j} T\right)^{1 / 6 j}\right\} \\
\ll & \left(U_{1} U_{2} W\right)^{-1} N_{1}^{1 / 2} T^{1 / 2-1 / 2 j} N_{2}^{1 / 4 j} \min \left\{L_{0}^{\frac{1}{2}}, T^{\frac{1}{2 j}}\right\} \\
& +\left(U_{1} U_{2} W\right)^{-1}\left(T N_{1}\right)^{1 / 2} \min \left(N_{2}^{1 / 4 j}, N_{2}^{1 / 12 j} L_{0}^{1 / 8}\right) .
\end{aligned}
$$


If $L_{0} \ll T^{1 / j}$, we have that

$$
L_{0}^{\frac{1}{2}} \ll T^{\frac{1}{25}} \text { and } N_{2}^{\frac{1}{65}} L_{0}^{\frac{1}{3}} \ll T^{\frac{1}{2 j}} .
$$

Thus the first term on the right hand side of (2.13) is less than the second term. If $L_{0} \gg T^{1 / j}$, by (2.7), we have $j=2$. We have that the first term on the right hand side of (2.13) is less than the second term again. Then

$$
F \ll\left(U_{1} U_{2} W\right)^{-1}\left(T N_{1}\right)^{1 / 2} \min \left(N_{2}^{1 / 4 j}, N_{2}^{1 / 12 j} L_{0}^{1 / 6}\right) .
$$

Thus (2.12) and (2.11) follows.

Case 3. $F \leq 2 U_{2}^{-2} N_{2}, F \geq 2 U_{1}^{-2} N_{1}$.

The proof here is the same as in Case 2.

Case 4. $F>2 U_{1}^{-2} N_{1}, F>2 U_{2}^{-2} N_{1}$.

We have that

$$
\begin{aligned}
F & \leq 2 \min \left\{U_{1}^{-2} T, U_{2}^{-2} T, U_{1}^{-6} N_{1} T, U_{2}^{-6} N_{2} T, W^{-2 j} L_{0}^{j}, W^{-2 j} T\right\} \\
& +2 \min \left\{U_{1}^{-2} T, U_{2}^{-2} T, U_{1}^{-6} N_{1} T, U_{2}^{-6} N_{2} T, W^{-6 j} L_{0}^{j}, W^{-2 j} T\right\} .
\end{aligned}
$$

Then

$$
\begin{aligned}
F & \ll\left(U_{1}^{-2} T\right)^{\frac{1}{2}}\left(W^{-2 j} L_{0}^{j}\right)^{\frac{1}{6 j}}\left(W^{-2 j} T\right)^{\frac{1}{3 j}}\left(U_{2}^{-2} T\right)^{\frac{1}{2}-\frac{3}{1 j}}\left(U_{2}^{-6} N_{2} T\right)^{\frac{1}{4 j}} \\
& +\left(U_{1}^{-2} T\right)^{\frac{1}{2}}\left(W^{-6 j} L_{0}^{j} T\right)^{\frac{1}{6 j}}\left(U_{2}^{-2} T\right)^{\frac{2 j-1}{6 j}}\left(U_{2}^{-6} N_{2} T\right)^{\frac{1}{12 j}} \\
& \ll\left(U_{1} U_{2} W\right)^{-1}\left(T^{1-\frac{1}{6 j}} L_{0}^{\frac{1}{6}} N_{2}^{\frac{1}{4 j}}+T L_{0}^{\frac{1}{6}} N_{2}^{\frac{1}{12 j}}\right) \\
& \leq\left(U_{1} U_{2} W\right)^{-1} T L_{0}^{\frac{1}{\delta}} N_{2}^{\frac{1}{12 j}},
\end{aligned}
$$

since $N_{2} \ll T$ by (2.7) with $j=1$. Thus we get (2.12) and (2.11) again.

Lemma 2.2. If $N_{2} \leq T$, we have

$$
U_{1} U_{2} W\left|S\left(U_{1}, U_{2}, W\right)\right| \ll x^{\frac{1}{2}-\delta}+x^{h_{1}^{i}\left(a_{1}, a_{2}\right)},
$$

where

$$
h_{1}\left(a_{1}, a_{2}\right)=\frac{i+1}{2(i+2)} t_{0}+\frac{1}{4(i+2)}+\frac{a_{1}^{\prime}+\sigma_{i}}{2}-\frac{a_{1}+\sigma}{4(i+2)},
$$


$a_{1}^{\prime}=\max \left\{t_{0}, \log N_{1} / \log x\right\}$ and $\sigma_{i}=\max \left\{\frac{t_{0}}{(i+2)}, 1-a_{1}-a_{2}\right\}$.

Proof. We consider two cases :

Case 1. $F \geq 2 U_{2}^{-2} N_{2}$

In this case, $N_{2} \ll T$, then

$$
\begin{aligned}
& F \ll \min \left\{U_{1}^{-2} x^{a_{1}^{i}}, U_{2}^{-2} T, U_{2}^{-6} N_{2} T, W^{-2(i+2)} x^{(i+2) \sigma_{i}}\right\} \\
& \ll\left(U_{1}^{-2} x^{a_{1}}\right)^{1 / 2}\left(W^{-2(i+2)} x^{(i+2) \sigma_{i}}\right)^{1 / 2(i+2)}\left(U_{2}^{-2} T\right)^{(2 i+1) / 4(i+2)}\left(U_{2}^{-6} N_{2} T\right)^{1 / 4(i+2)} \\
& \ll U_{1}^{-1} U_{2}^{-1} W^{-1} x^{\left(a_{1}^{\prime}+\sigma_{i}\right) / 2} T^{(i+1) / 2(i+2)} N_{2}^{1 / 4(i+2)} \\
& \ll U_{1}^{-1} U_{2}^{-1} W^{-1} x^{1 / 4(i+2)} x^{a_{1}^{\prime} / 2-a_{1} / 4(i+2)} T^{(i+1) / 2(i+2)} x^{\sigma_{i} / 2-\sigma / 4(i+2)},
\end{aligned}
$$

since $N_{2}=\boldsymbol{x} / N_{1} L_{0}=x^{1-a_{1}-\sigma}$.

Then (2.14) follows.

Case $2 \mathrm{~F}<2 U_{2}^{-2} \mathrm{~N}_{2}$

If $F<2 U_{1}^{-2} N_{1}$, the proof of this lemma is same as Case 1 of Lemma 2.1. If $F \geq 2 U_{1}^{-2} N_{1}$, then $N_{1} \leq T$. Interchanging $N_{1}$ with $N_{2}$, we may reduce this case to Case 1.

Lemma 2.3. Let $k_{0}=3$ and $\varepsilon<\sigma=1-a_{1}-a_{2}-a_{3} \leq t_{0} / 2$, then

$$
U_{1} U_{2} U_{3} W\left|S\left(U_{1}, U_{2}, U_{3}, W\right)\right| \ll x^{\frac{l}{2}-\delta}+x^{h_{2}\left(a_{1}, a_{2}, a_{3}\right)},
$$

where

$$
\begin{gathered}
h_{2}\left(a_{1}, a_{2}, a_{3}\right)=\frac{a_{1}^{\prime}}{2}+\frac{a_{2}^{\prime}+a_{3}^{\prime}}{2}+\frac{t_{0}}{6}+\frac{\sigma}{12}, \\
a_{1}^{\prime}=\frac{\log N_{1}^{\prime}}{\log x}=\max \left\{a_{1}, t_{0}\right\}, a_{2}^{\prime}=\frac{\log N_{2}^{\prime}}{\log x}=\max \left\{a_{2}, \frac{t_{0}}{3}\right\}
\end{gathered}
$$

and $a_{3}^{\prime}=\frac{\log N_{3}^{\prime}}{\log x}=\max \left\{a_{3}, \frac{t_{0}}{3}\right\}$.

Proof. In this case we have that $L_{0} \ll T^{1 / 2}$. Then

$$
\begin{gathered}
F \leq \min \left\{U_{1}^{-2}\left(N_{1}+T\right), U_{2}^{-6}\left(N_{2}^{3}+T\right), U_{3}^{-6}\left(N_{3}^{3}+T\right), W^{-4}\left(L_{0}^{2}+T\right),\right. \\
\left.W^{-4} L_{0}^{2}+W_{0}^{-12} L_{0}^{2} T, U_{3}^{-6} N_{3}^{3}+U_{3}^{-18} N_{3}^{3} T, U_{1}^{-2} N_{1}^{\prime}\right\} .
\end{gathered}
$$


We consider two cases :

Case 1. $F \leq 2 W^{-4} L_{0}^{2}$.

Suppose $F \leq 2 U_{3}^{-6} N_{3}^{3}$ and $F \leq 2 U_{2}^{-6} N_{2}^{3}$. By (2.4), we have that

$$
\begin{aligned}
F & \ll \min \left\{U_{1}^{-2} N_{1}, W^{-4} L_{0}^{2}, U_{2}^{-6} N_{2}^{3}, U_{3}^{-6} N_{3}^{3}\right\} \\
& \ll\left(U_{1}^{-2} N_{1}\right)^{\frac{1}{2}-\frac{1}{26}}\left(U_{2}^{-6} N_{2}^{3}\right)^{\frac{1}{8}-\frac{1}{78}}\left(U_{2}^{-6} N_{3}^{3}\right)^{\frac{1}{8}-\frac{1}{78}}\left(W^{-4} L_{0}^{2}\right)^{\frac{1}{4}-\frac{1}{32}} \\
& \ll\left(U_{1} U_{2} U_{3} W\right)^{-1+\frac{1}{13}}\left(N_{1} N_{2} N_{3} L_{0}\right)^{\frac{1}{2}-\frac{1}{26}} \ll x^{\frac{1}{2}-\delta} .
\end{aligned}
$$

Suppose $F>2 U_{3}^{-6} N_{3}^{3}$ and $F>2 U_{2}^{-6} N_{2}^{3}$, then

$$
N_{2} \ll T^{1 / 3}, N_{3} \ll T^{1 / 3} \text { and } F \ll U_{2}^{-18} N_{2}^{3} T,
$$

since $2 U_{i}^{-6} N_{i}^{3} \leq F \leq U_{i}^{-6}\left(N_{i}^{3}+T\right), F \leq U_{2}^{-18} N_{2}^{3} T+U_{2}^{-6} N_{2}^{3}$ for $i=$ 2 or $3, L_{0} \ll T^{1 / 2}$ and $N_{2} \ll T^{1 / 3}$. Therefore

$$
\begin{aligned}
F & \ll\left(U_{1}^{-2} N_{1}\right)^{\frac{1}{2}}\left(W^{-4} L_{0}^{2}\right)^{\frac{1}{4}}\left(U_{3}^{-6} T\right)^{\frac{1}{6}}\left(U_{2}^{-6} T\right)^{\frac{1}{24}}\left(U_{2}^{-18} N_{2}^{3} T\right)^{\frac{1}{24}} \\
& \ll\left(U_{1} U_{2} U_{3} W\right)^{-1} T^{\frac{1}{4}} N_{2}^{\frac{1}{8}} N_{1}^{\frac{1}{2}} L_{0}^{\frac{1}{2}} \\
& \ll\left(U_{1} U_{2} U_{3} W\right)^{-1} x^{h_{2}\left(a_{1}, a_{2}, a_{3}\right)}
\end{aligned}
$$

Suppose $F>2 U_{3}^{-6} N_{3}^{3}$ and $F \leq 2 U_{2}^{-6} N_{2}^{3}$, then

$$
\begin{aligned}
F & \ll\left(U_{1}^{-2} N_{1}\right)^{\frac{1}{2}}\left(U_{2}^{-6} N_{2}^{3}\right)^{\frac{1}{6}}\left(W^{-4} L_{0}^{2}\right)^{\frac{1}{4}}\left(U_{3}^{-6} T\right)^{\frac{1}{24}}\left(U_{3}^{-18} N_{3}^{3} T\right)^{\frac{1}{24}} \\
& \ll\left(U_{1} U_{2} U_{3} W\right)^{-1} N_{1}^{\frac{1}{2}} N_{2}^{\frac{1}{2}} L_{0}^{\frac{1}{2}} T^{\frac{1}{12}} N_{3}^{\frac{1}{8}} \ll\left(U_{1} U_{2} U_{3} W\right)^{-1} x^{h_{2}\left(a_{1}, a_{2}, a_{3}\right)}
\end{aligned}
$$

since $a_{3}^{\prime}=t_{0} / 3$ and $N_{3} \ll T^{1 / 3}$ in this case. Suppose $F<2 U_{3}^{-6} N_{3}^{3}$ and $F \geq 2 U_{2}^{-6} N_{2}^{3}$.

On interchanging $N_{2}$ with $N_{3}$, we may reduce this case to (2.18).

Case 2. $F>2 W^{-4} L_{0}^{2}$.

We have that

$$
F \ll\left(U_{1}^{-2} N_{1}\right)^{\frac{1}{2}}\left(U_{2}^{-6} N_{2}^{\prime 3}\right)^{\frac{1}{6}}\left(U_{3}^{-6} N_{3}^{\prime 3}\right)^{\frac{1}{6}}\left(W^{-4} T\right)^{\frac{1}{8}}\left(W^{-12} L_{0}^{2} T\right)^{\frac{1}{24}},
$$

then

$$
F \ll\left(U_{1} U_{2} U_{3} W\right)^{-1} N_{1}^{\frac{1}{2}} N_{2}^{\prime \frac{1}{2}} N_{3}^{\prime \frac{1}{2}} T^{\frac{1}{6}} L_{0}^{\frac{1}{12}} \ll\left(U_{1} U_{2} U_{3} W\right)^{-1} x^{h_{2}\left(a_{1}, a_{2}, a_{3}\right)} .
$$


The proof of Lemma 2.3 is now completed.

Lemma 2.4. If $k_{0}=3, a_{1} \geq t_{0}, a_{2} \geq t_{0} / 2$, then

$$
U_{1} U_{2} U_{3} W\left|S\left(U_{1}, U_{2}, U_{3}, W\right)\right| \ll x^{\frac{1}{2}-\delta}+x^{h_{3}\left(a_{1}, a_{2}, a_{3}\right)},
$$

where

$$
h_{3}\left(a_{1}, a_{2}, a_{3}\right)=\frac{a_{1}+a_{2}}{2}+\frac{a_{3}^{\prime}}{2}+\frac{t_{0}}{8}+\frac{\sigma}{6},
$$

$a_{3}^{\prime}=\log N_{3}^{\prime} / \log x=\max \left\{t_{0} / 4, a_{3}\right\}$ and $\sigma=1-a_{1}-a_{2}-a_{3}$.

Proof. We have that

$F \ll \min \left\{U_{1}^{-2} N_{1}, U_{2}^{-4} N_{2}^{2}, U_{3}^{-8}\left(N_{3}^{4}+T\right), W^{-6}\left(L_{0}^{3}+T\right), W^{-18} L_{0}^{3} T+W^{-6} L_{0}^{3}\right\}$

Suppose $F \leq 2 W^{-6} L_{0}^{3}$, we are in Case 1 of Lemma 2.1, so that

$$
U_{1} U_{2} U_{3} W F \ll x^{\frac{1}{2}-\delta} .
$$

Suppose $F>2 W^{-6} L_{0}^{3}$, then

$$
\begin{aligned}
F & \ll\left(U_{1}^{-2} N_{1}\right)^{\frac{1}{2}}\left(U_{2}^{-4} N_{2}^{2}\right)^{\frac{1}{4}}\left(U_{3}^{-8} x^{4 a_{3}^{\prime}}\right)^{\frac{1}{8}}\left(W^{-6} T\right)^{\frac{5}{18}}\left(W^{-18} L_{0}^{3} T\right)^{\frac{1}{)^{18}}} \\
& \ll\left(U_{1} U_{2} U_{3} W\right)^{-1} N_{1}^{\frac{1}{2}} N_{2}^{\frac{1}{2}} x^{\frac{a_{3}^{\prime}}{2}} T^{\frac{1}{8}} L_{0}^{\frac{1}{6}} \ll\left(U_{1} U_{2} U_{3} W\right)^{-1} x^{h_{3}\left(a_{1}, a_{2}, a_{3}\right) .}
\end{aligned}
$$

Thus the lemma follows.

§ 3. $L_{0}=L$.

In this section we discuss $\left|S\left(U_{1}, \cdots, U_{k}, W\right)\right|$ with (1.4), (1.5), (1.6), (1.7) and (1.9). We use the same notations as in $\S 2$ and assume $L_{0}=L$.

Lemma 3.1. Let $L=L_{0}$. If $L_{0} \geq T^{1 / 2+\varepsilon}$; then

$$
U_{1} \cdots U_{k_{0}} W\left|S\left(U_{1}, U_{k_{0}}, W\right)\right| \ll x^{\frac{1}{2}-\delta} .
$$

Proof. Take $j$ such that

$$
N_{1}^{j} \cdots N_{k_{0}}^{j} \geq T .
$$

We have

$$
F \ll \min \left\{U_{1}^{-2 j} \cdots U_{k_{0}}^{-2 j} N_{1}^{2 j}, W^{-4} T\right\}
$$


Thus

$$
\begin{aligned}
F & \ll\left(U_{1}^{-2 j} \cdots U_{k_{0}}^{-2 j} N_{1}^{2 j} \cdots N_{k_{0}}^{2 j}\right)^{3 / 4}\left(W^{-4} T\right)^{1 / 4} \\
& \ll\left(U_{1}^{-2 j} \cdots U_{k_{0}}^{-2 j} N_{1}^{2 j} \cdots N_{k_{0}}^{2 j}\right)^{1 / 2 j}\left(W^{-4} T\right)^{1 / 4} \\
& \ll\left(U_{1} \cdots U_{k_{0}} W\right)^{-1} N_{1}^{1 / 2} \cdots N_{k_{0}}^{1 / 2} T^{1 / 4},
\end{aligned}
$$

since $U_{1}^{-2 j} \cdots U_{k_{0}}^{-2 j} N_{1}^{2 j} \cdots N_{k_{0}}^{2 j} \leq 1$. We obtain

$$
U_{1} \ldots U_{k_{0}} W F \ll x^{1 / 2-\varepsilon / 2}
$$

by $L_{0} \geq T^{1 / 2+\varepsilon}$.

Lemma 3.2. $k_{0}=2, N_{1} \geq T, N_{2}<T^{1 / 5}$ and

$$
L_{0} N_{2}^{1 / 2} \geq T^{1 / 2+\varepsilon},
$$

then

$$
U_{1} U_{2} W\left|S\left(U_{1}, U_{2}, W\right)\right| \ll x^{\frac{1}{2}-\delta} .
$$

Proof. We have that, choosing $j$ such that $N_{2}^{j} \geq T$,

$$
F \ll \min \left\{W^{-4} U_{2}^{-2} T, U_{1}^{-2} N_{1}, U_{2}^{-j} N^{j}\right\} .
$$

Thus

$$
\begin{aligned}
F & \ll\left(W^{-4} U_{2}^{-2} T\right)^{1 / 4}\left(U_{1}^{-2} N_{1}\right)^{1 / 2}\left(U_{2}^{-2 j} N_{2}^{j}\right)^{1 / 4 j} \\
& \ll\left(U_{1} U_{2} W\right)^{-1} N_{1}^{1 / 2} N_{2}^{1 / 4} T^{1 / 4}=\left(U_{1} U_{2} W\right)^{-1}\left(x / L_{0} N_{2}\right)^{1 / 2} N_{2}^{1 / 4} T^{1 / 4} \\
& \ll x^{\frac{1}{2}-\delta}
\end{aligned}
$$

since $L_{0}^{1 / 2} N_{2}^{1 / 4} \geq T^{1 / 4+\varepsilon / 2}$.

Lemma 3.3. $k_{0}=2, N_{1} \geq T, N_{2} \geq T^{1 / 5}$ and

$$
L_{0} \geq T^{3 / 8+\varepsilon} N_{2}^{1 / 8},
$$

then

$$
U_{1} U_{2} W\left|S\left(U_{1}, U_{2}, W\right)\right| \ll x^{\frac{1}{2}-\delta} .
$$

Proof. We have that, choosing $j$ such that $N_{2}^{j} \geq T$,

$$
F \ll \min \left\{W^{-4} U_{2}^{-2} N_{2}^{5 / 4} T^{3 / 4}, U_{1}^{-2} N_{1}, U_{2}^{-2 j} N^{j}\right\} .
$$


Thus, by $U_{2}^{-2 j} N_{2}^{j} \leq 1$,

$$
\begin{aligned}
F & \ll\left(W^{-4} U_{2}^{-2} N_{2}^{5 / 4} T^{3 / 4}\right)^{1 / 4}\left(U_{1}^{-2} N_{1}\right)^{1 / 2}\left(U_{2}^{-2 j} N_{2}^{j}\right)^{1 / 4 j} \\
& \ll\left(U_{1} U_{2} W\right)^{-1} N_{1}^{1 / 2} N_{2}^{9 / 16} T^{3 / 16}=\left(U_{1} U_{2} W\right)^{-1}\left(x / L_{0} N_{2}\right)^{1 / 2} N_{2}^{9 / 16} T^{3 / 16} \\
& \ll x^{1 / 2-\delta} .
\end{aligned}
$$

Write

$$
\begin{gathered}
c_{1}=\frac{1}{2 j_{1}}+\frac{1}{2 j_{2}}-\frac{3}{10}, c_{2}=7+\frac{5}{j_{2}}+\frac{5}{j_{1}}, c_{3}=\frac{7}{8}-\frac{3}{4 j_{2}}-\frac{3}{4 j_{3}}-\frac{3}{8 j_{4}}, \\
c_{4}=\frac{1}{4}\left(\frac{1}{j_{2}}+\frac{1}{j_{3}}+\frac{1}{2 j_{4}}-\frac{1}{2}\right), c_{5}=\frac{3}{8}-\frac{1}{4 j_{2}}-\frac{3}{4 j_{3}}-\frac{3}{8 j_{4}},
\end{gathered}
$$

and

$$
c_{6}=\frac{1}{4 j_{2}}+\frac{1}{4 j_{3}}+\frac{1}{8 j_{4}}-\frac{1}{8} \text {. }
$$

Define

$$
N_{i}^{\prime}=\max \left\{N_{i}, T^{\frac{1}{j_{i}}}\right\}, i=3 \text { and } 4 .
$$

Lemma 3.4. Let $K_{0}=4, a_{3} \geq a_{4}, t_{0} / 3 \geq a_{4} \geq t_{0} / 5, \sigma=1-a_{1}-a_{2}-a_{3}-$ $a_{4} \geq t_{0} / j_{1}, j_{1}$ and $j_{2}, j_{3}, j_{4}$ be integers with $c_{i} \geq 0(1 \leq i \leq 6)$, then

$$
U_{1} U_{2} U_{3} U_{4} W\left|S\left(U_{1}, U_{2}, U_{3}, U_{4}, W\right)\right| \ll x^{\frac{1}{2}-\delta}+x^{h_{4}\left(a_{1}, a_{2}, a_{3}, a_{4}\right)}
$$

where

$$
\begin{gathered}
h_{4}\left(a_{1}, a_{2}, a_{3}, a_{4}\right)=\frac{a_{3}^{\prime}}{2}+\frac{a_{4}^{\prime}}{4}+\frac{5 a_{4}}{16}+\frac{3 t_{0}}{16} \\
+\max \left\{\frac{a_{1}}{2}+c_{6} j_{2} a_{2}+\left(c_{5}+c_{6}\right) t_{0}, c_{4} a_{1}+\frac{a_{2}}{2}+\left(c_{3}+c_{4}\right) t_{0}\right\} .
\end{gathered}
$$

Proof. We have that, by $t_{0} / 3 \geq a_{4} \geq t_{0} / 5, \sigma \geq t_{0} / 5$ and $a_{3} \geq a_{4}$,

$$
\begin{aligned}
F \ll & \min \left\{W^{-4} U_{4}^{-2} N_{4}^{\frac{5}{4}} T^{\frac{3}{4}}, U_{1}^{-2}\left(N_{1}+T\right), U_{1}^{-6} N_{1} T+U_{1}^{-2} N_{1}, U_{2}^{-2 j_{2}}\left(N_{2}^{j_{2}}+T\right)\right. \\
& U_{2}^{-2 j_{2}} N_{2}+U_{2}^{-6 j_{2}} N_{2}^{j_{2}} T, U_{3}^{-10} N_{3}^{5}, U_{4}^{-10} N_{4}^{5}, W^{-2 j_{1}} L_{0}^{j_{1}}, U_{3}^{-2 j_{3}} N_{3}^{\prime} j_{3}, \\
& \left.U_{4}^{-2 j_{4}}\left(N_{4}^{j_{4}}+T\right)\right\} .
\end{aligned}
$$


We discuss following cases

Case 1. $F \leq 2 U_{1}^{-2} N_{1}$ and $F \leq 2 U_{2}^{-2 j_{2}} N_{2}^{j_{2}}$.

By (2.4), we have that

$$
\begin{aligned}
F & \ll\left(U_{1}^{-2} N_{1}\right)^{\frac{1}{2}-\frac{\delta c_{1}}{c_{2}}}\left(U_{2}^{-2 j_{2}} N_{2}^{j_{2}}\right)^{\frac{1}{2 j_{2}}-\frac{s c_{1}}{j 2 c_{2}}}\left(U_{3}^{-10} N_{3}^{5}\right)^{\frac{1}{10}-\frac{c_{1}}{c_{2}}}\left(U_{4}^{-10} N_{4}^{5}\right)^{\frac{1}{10}-\frac{c_{1}}{c_{2}}} \times \\
& \times\left(W^{-2 j_{1}} L_{0}^{j_{1}}\right)^{\frac{1}{2 j_{1}}-\frac{s c_{1}}{j c_{2}}} \\
& \ll\left(U_{1} U_{2} U_{3} U_{4} W\right)^{-1+\frac{10 c_{1}}{c_{2}}}\left(N_{1} N_{2} N_{3} N_{4} L_{0}\right)^{\frac{1}{2}-\frac{\delta c_{1}}{c_{2}}} \ll\left(U_{1} U_{2} U_{3} U_{4} W\right)^{-1} x^{\frac{1}{2}-\delta},
\end{aligned}
$$

since $c_{1} / c_{2}<1 / 10$.

Case 2. $F>2 U_{1}^{-2} N_{1}$ and $F \leq 2 U_{2}^{-2 j_{2}} N_{2}^{j_{2}}$.

We use $F \ll U_{i}^{-2 j_{i}} N_{i}^{\prime j_{i}}$ for $i=3$ or 4 . We have that, by $2 c_{3}+6 c_{4}=1$,

$$
\begin{aligned}
F & \ll\left(W^{-4} U_{4}^{-2} N_{4}^{\frac{5}{4}} T^{\frac{3}{4}}\right)^{\frac{1}{4}}\left(U_{4}^{-2 j_{4}} N_{4}^{\prime j_{4}}\right)^{\frac{1}{j_{4}}}\left(U_{3}^{-2 j_{3}} N_{3}^{\prime j_{3}}\right)^{\frac{1}{2 j_{3}}}\left(U_{2}^{-2 j_{2}} N_{2}^{j_{2}}\right)^{\frac{1}{2 j_{2}}} \times \\
& \times\left(U_{1}^{-2} T\right)^{c_{3}}\left(U_{1}^{-6} N_{1} T\right)^{c_{4}} \\
& \ll\left(U_{1} U_{2} U_{3} U_{4} W\right)^{-1} N_{1}^{c_{4}} N_{2}^{\frac{1}{2}} N_{3}^{\frac{1}{2}} N_{4}^{\prime \frac{1}{4}} N_{4}^{\frac{5}{18}} T^{\frac{3}{16}}+c_{3}+c_{4} \\
& \ll\left(U_{1} U_{2} U_{3} U_{4} W\right)^{-1} x^{h_{4}\left(a_{1}, a_{2}, a_{3}, a_{4}\right)},
\end{aligned}
$$

Case 3. $F \leq 2 U_{1}^{-2} N_{1}$ and $F>2 U_{2}^{-2 j_{2}} N_{2}^{j_{2}}$.

Then we have that

$$
\begin{aligned}
F & \ll\left(W^{-4} U_{4}^{-2} N_{4}^{\frac{5}{4}} T^{\frac{3}{4}}\right)^{\frac{1}{4}}\left(U_{4}^{-2 j_{4}} N_{4}^{\prime j_{4}}\right)^{\frac{1}{j_{1}}}\left(U_{3}^{-2 j_{3}} N_{3}^{\prime j_{3}}\right)^{\frac{1}{2 j_{3}}}\left(U_{1}^{-2} N_{1}\right)^{\frac{1}{2}} \times \\
& \times\left(U_{2}^{-2 j_{2}} T\right)^{c_{5}}\left(U_{2}^{-6 j_{2}} N_{2}^{j_{2}} T\right)^{c_{6}} \\
& \ll\left(U_{1} U_{2} U_{3} U_{4} W\right)^{-1} N_{1}^{\frac{1}{2}} N_{2}^{j_{2} c_{6}} N_{3}^{\prime \frac{1}{2}} N_{4}^{\frac{1}{4}} N_{4}^{\frac{5}{16}} T^{\frac{3}{16}}+c_{5}+c_{8} \\
& \ll\left(U_{1} U_{2} U_{3} U_{4} W\right)^{-1} x^{h_{4}\left(a_{1}, a_{2}, a_{3}, a_{4}\right)},
\end{aligned}
$$

as required.

Case 4. $F>2 U_{1}^{-2} N_{1}$ and $F>2 U_{2}^{-2 j_{2}} N_{2}^{j_{2}}$.

In this case we have that $N_{1} \ll T$ and $N_{2} \ll T^{1 / \dot{\gamma}_{3}}$. Thus (3.3) and (3.4) are true again, i.e. we get (3.1) again. 
If take $j_{2}=2$ and $j_{3}=j_{4}=5$, then

$h_{4}\left(a_{1}, a_{2}, a_{3}, a_{4}\right)=\frac{a_{3}^{\prime}}{2}+\frac{a_{4}^{\prime}}{4}+\frac{5 a_{4}}{16}+\frac{3 t_{0}}{16}+\max \left\{\frac{a_{1}}{2}+\frac{3}{20} a_{2}+\frac{t_{0}}{10}, \frac{3}{40} a_{1}+\frac{a_{2}}{2}+\frac{7}{20} t_{0}\right\}$

Lemma 3.5. Suppose $k_{0}=4, a_{4} \leq t_{0} / 5, a_{i} \geq t_{0} / j_{i},(i=2,3$ and 4$)$ and $\sigma \geq t_{0} / j$ with

$$
\frac{1}{j_{2}}+\frac{1}{j_{3}}+\frac{1}{j_{4}}+\frac{1}{j}>\frac{1}{2}
$$

then

$$
U_{1} U_{2} U_{3} U_{4} W\left|S\left(U_{1}, U_{2}, U_{3}, U_{4}, W\right)\right|<x^{\frac{1}{2}-\delta}+x^{h_{5}\left(a_{1}, a_{2}, a_{3}, a_{4}\right)}
$$

where

$$
\begin{gathered}
h_{5}\left(a_{1}, a_{2}, a_{3}, a_{4}\right)=\frac{a_{3}^{\prime}}{2}+\frac{a_{4}^{\prime}}{4}+\frac{t_{0}}{4}+ \\
\max \left\{\frac{a_{1}}{2}+c_{6} j_{2} a_{2}+\left(c_{5}+c_{6}\right) t_{0}, a_{1}+\frac{a_{2}}{2}+\left(c_{3}+c_{4}\right) t_{0}\right\} .
\end{gathered}
$$

Proof. In the proof of Lemma 3.2 we replace (1.10) by (1.11). Then we replace $5 a_{4} / 16$ by $t_{0} / 16$ and we get $h_{5}\left(a_{1}, a_{2}, a_{3}, a_{4}\right)$ replace $h_{4}\left(a_{1}, a_{2}, a_{3}, a_{4}\right)$. The lemma follows.

Lemma 3.6. If $k_{0}=3, a_{1} \geq t_{0}$ and $t_{0} / 3 \geq a_{3} \geq t_{0} / 5$, then

$$
U_{1} U_{2} U_{3} W\left|S\left(U_{1}, U_{2}, U_{3}, W\right)\right| \ll x^{\frac{1}{2}-\delta}+x^{h_{6}\left(a_{1}, a_{2}, a_{3}\right)}
$$

where

and $a_{2}^{\prime}=\max \left\{a_{2}, t_{0} / 3\right\}$.

$$
h_{6}\left(a_{1}, a_{2}, a_{3}\right)=\frac{13 t_{0}}{48}+\frac{a_{1}+a_{2}^{\prime}}{2}+\frac{5 a_{3}}{16} .
$$

Proof. We have

$$
\begin{aligned}
F & \ll\left(W^{-4} U_{3}^{-2} N_{3}^{\frac{5}{4}} T^{\frac{3}{4}}\right)^{\frac{1}{4}}\left(U_{1}^{-2} N_{1}\right)^{\frac{1}{2}}\left(U_{2}^{-6} x^{3 \alpha_{2}^{\prime}}\right)^{\frac{1}{6}}\left(U_{3}^{-6} T\right)^{\frac{1}{12}} \\
& \ll\left(U_{1} U_{2} U_{3} W\right)^{-1} N_{1}^{\frac{1}{2}} N_{3}^{\frac{5}{16}} x^{\frac{-2}{2}} T^{\frac{13}{6}} \ll\left(U_{1} U_{2} U_{3} W\right)^{-1} x^{h_{6}\left(a_{1}, a_{2}, a_{3}, a_{4}\right) .}
\end{aligned}
$$

Thus the lemma follows.

Lemma 3.7. If $k_{0}=4$ and the $a_{i}$ 's satisfy: (1) $a_{1}+a_{3}+a_{4} \geq t_{0}$; (2) 
$a_{2}+a_{3}+a_{4} \leq t_{0} ;(3) a_{1}+a_{3} \leq t_{0}$, (4) $a_{1}+a_{4} \leq t_{0} ;$ and (5) $a_{4} \leq a_{3} \leq t_{0} / 5$

then

$$
U_{1} U_{2} U_{3} U_{4} W\left|S\left(U_{1}, U_{2}, U_{3}, U_{4}, W\right)\right|<x^{\frac{1}{2}-\delta}+x^{h_{7}\left(a_{1}, a_{2}, a_{3}, a_{4}\right)},
$$

where

$$
h_{7}\left(a_{1}, a_{2}, a_{3}, a_{4}\right)=t_{0}+a_{2} / 8 \text {. }
$$

Proof. W.L.O.G. we can agree that $U_{3} \geq U_{4}$, then we have

$F \leq \min \left\{W^{-4} U_{3}^{-2} T, U_{1}^{-2} U_{3}^{-2} T, U_{2}^{-2} U_{3}^{-2} U_{4}^{-2} T, U_{2}^{-2} N_{2}+U_{2}^{-6} N_{2} T, U_{1}^{-2} U_{3}^{-2} U_{4}^{-2} N_{1} N_{3} N_{4}\right\}$.

If $F \leq 2 U_{2}^{-2} N_{2}$, this is Case 1 of Lemma 2.1 ; if $F \geq 2 U_{2}^{-2} N_{2}$, from $U_{3} \geq U_{4}$, we have

$$
\begin{aligned}
F & \ll\left(W^{-4} U_{3}^{-2} T\right)^{\frac{1}{4}}\left(U_{1}^{-2} U_{3}^{-2} T\right)^{\frac{1}{2}}\left(U_{2}^{-2} U_{3}^{-2} U_{4}^{-2} T\right)^{\frac{1}{8}}\left(U_{2}^{-6} N_{2} T\right)^{\frac{1}{8}} \\
& \ll\left(U_{1} U_{2} U_{3} U_{4} W\right)^{-1} T N_{2}^{\frac{1}{8}} U_{3}^{-\frac{3}{4}} U_{4}^{\frac{3}{3}} \ll\left(U_{1} U_{2} U_{3} U_{4} W\right)^{-1} x^{h_{7}\left(a_{1}, a_{2}, a_{3}, a_{4}\right)},
\end{aligned}
$$

and the lemma follows.

Lemma 3.8. Suppose that $k_{0}=6, t_{0} / 3 \geq a_{1} \geq \cdots \geq a_{6} \geq t_{0} / 5$ and $\sigma+a_{1}+a_{2} \geq t_{0}$, then

$$
U_{1} U_{2} \cdots U_{6} W\left|S\left(U_{1}, \cdots, U_{6}\right)\right| \ll x^{\frac{1}{2}-\delta}+x^{h_{8}\left(a_{1}, \cdots, a_{6}\right)}
$$

where

$$
h_{8}\left(a_{1}, \cdots, a_{6}\right)=\frac{19}{48} a_{6}+\frac{a_{5}}{12}+\frac{a_{4}}{12}+\frac{15}{16} t_{0} .
$$

Proof. If $F \leq U_{6}^{-2} U_{5}^{-2} U_{4}^{-2} N_{6} N_{5} N_{4}$, then

$$
\begin{aligned}
F & \ll\left(U_{6}^{-2} U_{5}^{-2} U_{4}^{-2} N_{6} N_{5} N_{4}\right)^{\frac{1}{2}-\frac{1}{22}}\left(W^{-2} U_{1}^{-2} U_{2}^{-2} L_{0} N_{1} N_{2}\right)^{\frac{1}{2}-\frac{1}{22}}\left(U_{3}^{-10} N_{3}^{5}\right)^{\frac{1}{10}-\frac{1}{110}} \\
& \ll\left(W U_{6} \cdots U_{1}\right)^{-1+\frac{1}{11}}\left(L_{0} N_{6} \cdots N_{1}\right)^{\frac{1}{2}-\frac{1}{22}} \ll x^{\frac{1}{2}-\delta} .
\end{aligned}
$$

If $F>U_{6}^{-2} U_{5}^{-2} U_{4}^{-2} N_{6} N_{5} N_{4}$, then

$$
F \leq U_{6}^{-6} U_{5}^{-6} U_{4}^{-6} N_{6} N_{5} N_{4} T,
$$


and

$$
\begin{aligned}
F & \ll\left(W^{-4} U_{6}^{-2} N_{6}^{\frac{5}{2}} T^{\frac{3}{4}}\right)^{\frac{1}{4}}\left(U_{1}^{-2} U_{2}^{-2} U_{3}^{-2} T\right)^{\frac{1}{2}}\left(U_{4}^{-6} U_{5}^{-6} U_{6}^{-6} N_{4} N_{5} N_{6} T\right)^{\frac{1}{12}} \times \\
& \times\left(U_{4}^{-4} U_{5}^{-2} T\right)^{\frac{1}{3}}\left(U_{5}^{-6} T\right)^{\frac{1}{24}} \\
& \ll\left(W U_{1} \cdots U_{6}\right)^{-1} T^{\frac{15}{16}} N_{4}^{\frac{1}{12}} N_{5}^{\frac{1}{12}} N_{6}^{\frac{19}{18}} \ll\left(W U_{1} \cdots U_{6}\right)^{-1} x^{h_{8}\left(a_{1}, \cdots, \alpha_{6}\right)} .
\end{aligned}
$$

Lemma 3.9. Suppose that $k_{0}=4, a_{1} \geq a_{2} \geq a_{3} \geq a_{4}, a_{1}+a_{3} \leq t_{0}<$ $a_{1}+a_{3}+a_{4}, \sigma \geq t_{0} / 3$ and $2 a_{4} \leq t_{0} / 3$, then

$$
U_{1} \cdots U_{4} W\left|S\left(U_{1}, \cdots, U_{4}\right)\right| \ll x^{\frac{1}{2}-\delta}+x^{h_{9}\left(a_{1}, \cdots, a_{4}\right)}
$$

where

$$
h_{9}\left(a_{1}, \cdots, a_{4}\right)=\frac{15}{16} t_{0}+\frac{a_{2}}{8}+\frac{5 a_{4}}{8} .
$$

Proof. If $F \leq 2 U_{2}^{-2} N_{2}$, then

$$
F \ll\left(U_{1}^{-2} U_{3}^{-2} U_{4}^{2} N_{1} N_{3} N_{4}\right)^{\frac{1}{2}-\frac{1}{30}}\left(U_{2}^{-2} N_{2}\right)^{\frac{1}{2}-\frac{1}{30}}\left(W^{-6} L_{0}^{3}\right)^{\frac{1}{6}-\frac{1}{10}} \ll x^{\frac{1}{2}-\delta}
$$

by (2.4).

If $F>2 U_{2}^{-2} N_{2}$, then $F \ll \min \left\{U_{2}^{-2} T, U_{2}^{-6} N_{2} T\right\}$. Thus

$$
\begin{aligned}
F & \ll\left(W^{-4} U_{4}^{-4} N_{4}^{\frac{5}{2}} T^{\frac{3}{4}}\right)^{\frac{1}{4}}\left(U_{1}^{-2} U_{3}^{-2} T\right)^{\frac{1}{2}}\left(U_{2}^{-2} T\right)^{\frac{1}{5}}\left(U_{2}^{-6} N_{2} T\right)^{\frac{1}{8}} \\
& \ll T^{\frac{15}{16}} N_{2}^{\frac{1}{8}} N_{4}^{\frac{5}{8}} \ll x^{h_{9}\left(a_{1}, \cdots, a_{4}\right)},
\end{aligned}
$$

as required.

Lemma 3.10. Suppose that $k_{0}=3, a_{2}+a_{3} \leq t_{0}, a_{1}+\sigma \geq t_{0}$ and $a_{3} \leq t_{0} / 5$, then

$$
U_{1} \cdots U_{4} W\left|S\left(U_{1}, \cdots, U_{4}\right)\right| \ll x^{\frac{1}{2}-6}+x^{h_{10}\left(a_{1}, \cdots, a_{4}\right)}
$$

where

$$
h_{10}\left(a_{1}, \cdots, a_{4}\right)=t_{0}+\frac{a_{2}}{8}+\frac{a_{3}}{24} .
$$

Proof. We have that

$$
F \ll \min \left\{W^{-4} U_{3}^{-2} T, U_{1}^{-2} T, U_{2}^{-2} U_{3}^{-2}\left(T+N_{2} N_{3}\right), U_{2}^{-6} U_{3}^{-6} N_{2} N_{3} T+U_{2}^{-2} U_{3}^{-2} N_{2} N_{3}\right.
$$




$$
\left.W^{-2} U_{1}^{-2} L_{0} N_{1}\right\}
$$

If $F \leq 2 U_{2}^{-2} U_{3}^{-2} N_{2} N_{3}$, then we go back to the Case 1 on Lemma 3.1, since $F \ll W^{-2} U_{1}^{-2} L_{0} N_{1}$, and $N_{4} \geq x^{\varepsilon} ;$ if $F>2 U_{2}^{-2} U_{3}^{-2} N_{2} N_{3}$, then

$$
\begin{aligned}
F & \ll\left(W^{-4} U_{3}^{-2} T\right)^{\frac{1}{4}}\left(U_{1}{ }^{2} T\right)^{\frac{1}{2}}\left(U_{2}^{-2} U_{3}^{-2} T\right)^{\frac{1}{3}}\left(U_{2}^{-6} U_{3}^{-6} N_{2} N_{3} T\right)^{\frac{1}{23}}\left(U_{2}^{-6} N_{2} T\right)^{\frac{1}{12}} \\
& \ll\left(U_{1} U_{2} U_{3} W\right)^{-1} x^{h_{10}\left(a_{1}, a_{2}, a_{3}\right)},
\end{aligned}
$$

as required.

§4. SET $E(\theta)$.

Let $\theta_{j}(1 \leq j \leq r)$ be positive numbers with

$$
\sum_{1 \leq i \leq r} \theta_{i}<1
$$

Denote $\left\{\theta_{j}\right\}=\left\{\theta_{0}, \theta_{1}, \cdots, \theta_{r}\right\}$ and

$$
\theta_{0}=1-\sum_{1 \leq i \leq r} \theta_{i}
$$

Divide the set of numbers

$$
\left\{\theta_{0}, \theta_{1}, \cdots, \theta_{r}\right\}
$$

into three subsets and call the sums of terms in each subset $a_{1}, a_{2}$ and $\sigma$, where $\sigma$ is distinguished by being $\theta_{0}$ if $\sigma>t_{0} / 2$, and otherwise $\sigma \leq t_{0} / 2$. Since $a_{1}+a_{2}+\sigma=1$, any two of $a_{1}, a_{2}, \sigma$ determine the third. We attach an exactly similar meaning to $\left\{a_{1}, a_{2}, a_{3}, \sigma\right\}$ and $\left\{a_{1}, a_{2}, a_{3}, a_{4}, \sigma\right\}$. We refer to $\left\{a_{1}, a_{2}, \sigma\right\}$, or $\left\{a_{1}, a_{2}, a_{3}, \sigma\right\}$, or $\left\{a_{1}, a_{2}, a_{3}, a_{4}, \sigma\right\}$ as set of complementary partial sums. For $\left\{a_{1}, \cdots, a_{k}, \sigma\right\}$ if there exists some $h_{i}\left(a_{1}, \cdots, a_{k}\right)$ which satisfies (1.8) with

$$
h_{i}\left(a_{1}, \cdots, a_{k}\right)<1 / 2 \text {, }
$$

then we call it $\left\{a_{1}, \cdots, a_{k}, \sigma\right\} \in E(\theta)$. For short, we write that $\left\{\theta_{j}\right\} \in E(\theta)$ instead of $\left\{a_{1}, \cdots, a_{k}, \sigma\right\} \in E(\theta)$.

Lemma 4.1. If there exists at least one set of complementary partial sums 
$\left\{a_{1}, a_{2}, \sigma\right\}$ (or $\left.\left\{a_{1}, a_{2}, a_{3}, \sigma\right\}\right)$ of $\left\{\theta_{j}\right\}$ such that at least one of conditions (4.1.1) - (4.1.4) (or (4.1.5)) holds, then $\left\{\theta_{j}\right\} \in E(\theta)$.

(4.1.1) $a_{1} \geq a_{2} \geq t_{0}$ and $\sigma>\varepsilon$;

This situation is covered by (2.9) and (2.10) of Lemma 2.1

(4.1.2) $a_{2} \leq a_{1} \leq t_{0}$ and $a_{2}<4-8 t_{0}$;

Using (2.11) of Lemma 2.1 with $a_{1}^{\prime}=t_{0}$ and $j=2$, we have that

$$
h_{0}\left(a_{1}, a_{2}\right)=t_{0}+\min \left\{a_{2} / 8, \sigma / 6+a_{2} / 24\right\} \leq t_{0}+a_{2} / 8<1 / 2 .
$$

(4.1.3) $a_{1} \geq t_{0}, a_{2}>\frac{2 i+2}{2 i+3} t_{0}$ and $\sigma \geq \frac{t_{0}}{i+2} ;$

In this case, we use Lemma 2.2, $\sigma_{i}=\max \left\{t_{0} /(i+2), 1-a_{1}-a_{2}\right\}=$ $\max \left\{t_{0} /(i+2), \sigma\right\}=\sigma$, and $a_{1}^{\prime}=a_{1}$, then

$$
\begin{aligned}
h_{1}^{i}\left(a_{1}, a_{2}\right) & =\frac{i+1}{2(i+2)} t_{0}+\frac{1}{4(i+2)}+\frac{2 i+3}{4(i+2)}\left(a_{1}+\sigma\right) \\
& =\frac{i+1}{2(i+2)} t_{0}+\frac{1}{4(i+2)}+\frac{2 i+3}{4(i+2)}\left(1-a_{2}\right)<\frac{1}{2} .
\end{aligned}
$$

(4.1.4) $a_{3} \leq a_{2} \leq t_{0} / 3 \leq a_{1} \leq t_{0}, \sigma=1-a_{1}-a_{2}-a_{3}$ and $a_{1}+\sigma / 6<1-t_{0}$.

By Lemma 2.3 with $a_{2}^{\prime}=\max \left\{t_{0} / 3, a_{2}\right\}=t_{0} / 3, a_{3}^{\prime}=\max \left\{t_{0} / 3, a_{3}\right\}=$ $t_{0} / 3 ;$ then

$$
\begin{aligned}
h_{2}\left(a_{1}, a_{2}, a_{3}\right) & =a_{1} / 2+\left(a_{2}^{\prime}+a_{3}^{\prime}\right) / 2+t_{0} / 6+\sigma / 12 \\
& =a_{1} / 2+t_{0} / 2+\sigma / 12<1 / 2 .
\end{aligned}
$$

Lemma 4.2. If there exists at least one set of complementary partial sums $\left\{a_{1}, a_{2}, a_{3}, \sigma\right\}$ of $\left\{\theta_{j}\right\}$ such that at least one of conditions (4.2.1) - (4.2.3) holds, then $\left\{\theta_{j}\right\} \in E(\theta)$.

(4.2.1). $a_{1} \geq t_{0}, a_{2} \geq t_{0} / 2, a_{3} \geq t_{0} / 4$ and $\sigma>2 t_{0} / 7$.

In this case, Lemma 2.4 is applied, we have $a_{3}^{\prime}=\max \left\{a_{3}, t_{0} / 4\right\}=a_{3}$, then

$$
\begin{aligned}
h_{3}\left(a_{1}, a_{2}, a_{3}\right) & =\left(a_{1}+a_{2}+a_{3}\right) / 2+t_{0} / 8+\sigma / 16 \\
& =1 / 2+t_{0} / 8-7 \sigma / 16<1 / 2,
\end{aligned}
$$

since $\sigma>2 t_{0} / 7$.

(4.2.2). $a_{1} \geq t_{0}, a_{2} \geq \hat{a}_{3} \geq t_{0} / 3$ and $\sigma>2 t_{0} / 5$. 
Using Lemma 2.3, we have $a_{2}^{\prime}=\max \left\{t_{0} / 3, a_{2}\right\}=a_{2}$ and $a_{3}^{\prime}=\max \left\{t_{0} / 3, a_{3}\right\}=$ $a_{3}$, then

$$
\begin{aligned}
h_{3}\left(a_{1}, a_{2}, a_{3}\right) & =\left(a_{1}+a_{2}+a_{3}\right) / 2+t_{0} / 6+\sigma / 12 \\
& =(1-\sigma) / 2+t_{0} / 6+\sigma / 12 \\
& =1 / 2+t_{0} / 6-5 \sigma / 12<1 / 2
\end{aligned}
$$

since $\sigma>2 t_{0} / 5$.

(4.2.3). $a_{1} \geq t_{0}, a_{2} \geq t_{0} / 3 \geq a_{3}$ and $t_{0} / 2 \geq \sigma>\max \left\{2 t_{0} / 5,4 t_{0} / 5-6 a_{3} / 5\right\}$.

We use Lemma 2.3 with $a_{2}^{\prime}=\max \left\{t_{0} / 3, a_{2}\right\}=a_{2}$, and $a_{3}^{\prime}=\max \left\{t_{0} / 3, a_{3}\right\}=$ $t_{0} / 3$, then

$$
\begin{aligned}
h_{3}\left(a_{1}, a_{2}, a_{3}\right) & =\left(a_{1}+a_{2}\right) / 2+t_{0} / 6+t_{0} / 6+\sigma / 12 \\
& =\left(1-\sigma-a_{3}\right) / 2+t_{0} / 3+\sigma / 12 \\
& =1 / 2+t_{0} / 3-5 \sigma / 12-a_{3} / 3<1 / 2
\end{aligned}
$$

since $\sigma>4 t_{0} / 5-6 a_{3} / 5$.

Lemma 4.3. Suppose $L=L_{0}$ (i.e. (1.9) holds). If $\sigma>t_{0} / 2$; then $\left\{\theta_{j}\right\} \in$ $E(\theta)$.

It is covered by Lemma 3.1 .

For a fixed $\sigma$, denote

$$
M_{\sigma}=\sup _{\substack{\left\{a_{1}, a_{2}, \sigma_{0} \in\left\{(\theta) \\ a_{2} \leq t_{0} \leq a_{1}\right.\right.}}\left\{a_{1}\right\} \text { and } m_{\sigma}=\inf _{\substack{\left\{a_{1}, a_{2}, \sigma\right\} \in\{\theta\} \\ a_{2} \leq t_{0} \leq a_{1}}}\left\{a_{2}\right\}
$$

Lemma 4.4. Suppose that $\left\{\theta_{j}\right\}$ satisfies the condition (4.4.1): (4.4.1) all of complementary partial sums $\left\{a_{1}, a_{2}, \sigma\right\}$ with $a_{2} \leq a_{1} \leq t_{0}$ satisfies $\left\{\theta_{j}\right\} \in E(\theta),\left(a_{2} \leq a_{1}\right.$ is not necessary). Then for a fixed $\sigma,\left\{\theta_{j}\right\} \in E(\theta)$ if

$$
m_{\sigma}<a_{2}<M_{\sigma}
$$

Moreover, we have that

$$
m_{\sigma}+a_{2}+M_{\sigma}=1
$$




$$
m_{\sigma}<t_{0}<M_{\sigma}
$$

for $t_{0} / j \geq \sigma \geq 2 t_{0} /(2 j+1)$,

$$
\begin{gathered}
M_{\sigma} \geq 1-\frac{6 j}{6 j-1} t_{0}-\frac{2 j-1}{6 j-1} \sigma \\
m_{\sigma} \leq \frac{6 j}{6 j-1} t_{0}-\frac{4 j}{6 j-1} \sigma
\end{gathered}
$$

and for $2 t_{0} /(2 j+1) \geq \sigma \geq t_{0} /(j+1)$,

$$
\begin{gathered}
M_{\sigma} \geq 1-\frac{2 j}{2 j+1} t_{0}-\sigma, \\
m_{\sigma} \leq \frac{2 j}{2 j+1} t_{0} .
\end{gathered}
$$

Proof. We use (4.13) with $j=i+1$ to prove (4.4.8). Then we have (4.4.7) by (4.4.3).

For $\theta>11 / 20$; we can get much simple conditions for $\left\{a_{1}, a_{2}, \sigma\right\} \in E(\theta)$. We will use it to discuss the gap between consecutive primes in [3].

Lemma 4.5. Suppose that $t_{0}<9 / 20$. If there exists at least one set of complementary partial sums $\left\{a_{1}, a_{2}, \sigma\right\}$ with $a_{1} \leq t_{0}$, and $a_{2} \leq t_{0}$, then $\left\{\theta_{j}\right\} \in E(\theta)$. Moreover, we have that

$$
M_{\sigma}-m_{\sigma}>\sigma, \text { if } \sigma<0.1
$$

and

$$
M_{\sigma}-m_{\sigma}>1-2 t_{0} \text {, if } \sigma \geq 0.1 \text {. }
$$

Proof. If $a_{2} \leq a_{1}$, and $a_{2}<4-8 t_{0}$, it is same as (5.1.8). If $a_{1} \geq a_{2} \geq 4-8 t_{0}$, using (2.11) of Lemma 2.1 with $j=2$, we have

$$
\begin{aligned}
h_{0}\left(a_{1}, a_{2}\right) & =t_{0}+\min \left\{a_{2} / 8, \sigma / 6+a_{2} / 24\right\} \\
& \leq t_{0}+\left(4-8 t_{0}\right) / 24+\left(1-2\left(4-8 t_{0}\right)\right) / 6<1 / 2 .
\end{aligned}
$$

By (4.4.4), (4.4.5) and (4.4.6), we have that

$$
M_{\sigma}-m_{\sigma} \geq 1-\frac{28}{29} t_{0}-\frac{9}{29} \sigma-\left(\frac{28}{29} t_{0}-\frac{20}{29} t_{0}\right) \geq \sigma,
$$


if $\sigma<t_{0} / 5$. When $t_{0} / 5 \leq \sigma<0.1$, we have that

$$
M_{\sigma}-m_{\sigma} \geq 1-\frac{8}{9} t_{0}-\sigma-\frac{8}{9} t_{0}>1-2 t_{0} .
$$

Then (4.5.1) holds. When $t_{0} /(j+1) \leq \sigma \leq 2 t_{0} /(2 j+1)$, by (4.4.4) and (4.4.6),

$$
M_{\sigma}-m_{\sigma} \geq 1-\frac{2 j}{2 j+1} t_{0}-\sigma-\frac{2 j}{2 j+1} t_{0} \geq 1-2 t_{0} .
$$

When $2 t_{0} /(2 j+1) \leq \sigma \leq t_{0} /(j+1)$, by (4.4.5) and (4.4.7)

$$
M_{\sigma}-m_{\sigma} \geq 1-\frac{6 j}{6 j-1} t_{0}-\frac{2 j-1}{6 j-1} \sigma-\left(\frac{6 j}{6 j-1} t_{0}^{\prime}-\frac{4 j}{6 j-1} \sigma\right) \geq 1-2 t_{0}
$$

Thus (4.5.2) holds.

For $\theta>6 / 11$, we can get some simple conditions for $\left\{a_{1}, a_{2}, \sigma\right\} \in E(\theta)$. We will use it to discuss the gap between consecutive primes in [3]. Moreover, we have that

Corollary 4.5.1. Suppose that $\theta>6 / 11$. (4.5.1) and (4.5.2) hold. If $a_{2} \leq$ $a_{1} \leq 1 / 2$ and $\sigma=1-a_{1}-a_{2}<1 / 2-8 t_{0} / 9$, then $\left\{\theta_{j}\right\} \in E(\theta)$.

Proof. If $a_{2} \geq t_{0}$, it is covered by (4.1.1); if $a_{1}<t_{0}$, we have that $\left\{\theta_{j}\right\} \in$ $E(\theta)$ by (4.5.1); if $a_{2} \leq t_{0}<a_{1}<1 / 2$ and $\sigma \geq t_{0} / 5$, then

$$
a_{2}=1-a_{1}-\sigma>1-1 / 2-\left(1 / 2-8 t_{0} / 9\right)=8 t_{0} / 9
$$

thus $\left\{\theta_{j}\right\} \in E(\theta)$ by (4.1.3). If $a_{2} \leq t_{0}<a_{1}<1 / 2$ and $\sigma<t_{0} / 5$, using (2.11) in Lemma 2.1 with $j=5$, then

$$
\begin{aligned}
h_{0}\left(a_{1}, a_{2}\right) & =t_{0} / 2+a_{1} / 2+\min \left\{a_{2} / 8, \sigma / 6+a_{2} / 60\right\} \\
& \leq t_{0} / 2+a_{1} / 2+\sigma / 6+a_{2} / 60 \\
& =t_{0} / 2+a_{1} / 2+\sigma / 6+\left(1-a_{1}-\sigma\right) / 60 \\
& =t_{0} / 2+1 / 60+29 a_{1} / 60+3 \sigma / 20 \\
& \leq t_{0} / 2+1 / 60+29 / 120+3 t_{0} / 100<1 / 2
\end{aligned}
$$


since $a_{1} \leq 1 / 2$ and $\sigma \leq t_{0} / 5$.

Lemma 4.6. Suppose $t_{0}<5 / 11$. If there exists at least one set of complementary partial sums $\left\{a_{1}, a_{2}, \sigma\right\}$ (or $\left\{a_{1}, a_{2}, a_{3}, \sigma\right\}$ ) of $\left\{\theta_{j}\right\}$ such that at least one of conditions (4.6.1) - (4.6.5) holds, then $\left\{\theta_{j}\right\} \in E(\theta)$.

(4.6.1) $a_{2} \leq a_{1} \leq t_{0}$ and $\sigma<1-20 t_{0} / 11$.

Proof. When $\sigma \geq t_{0} / 3$, we apply Lemma 2.2 with $i=1$,

$\sigma_{1}=\max \left\{t_{0} / 3,1-a_{1}-a_{2}\right\}=\max \left\{t_{0} / 3, \sigma\right\}=\sigma$ and $a_{1}^{\prime}=t_{0}$, then

$$
\begin{aligned}
h_{1}^{i}\left(a_{1}, a_{2}\right) & =t_{0} / 3+1 / 12+t_{0} / 2+\sigma / 2-\left(a_{1}+\sigma\right) / 12 \\
& \leq 5 t_{0} / 6+1 / 12+5 \sigma / 12-a_{1} / 12 \\
& \leq 5 t_{0} / 6+1 / 24+11 \sigma / 24 \\
& <5 t_{0} / 6+1 / 24+11\left(1-20 t_{0} / 11\right) / 24=1 / 2
\end{aligned}
$$

since $a_{1} \geq(1-\sigma) / 2 .\left(a_{1} \geq a_{2}\right.$ and $\left.a_{1}+a_{2}+\sigma=1\right)$.

When $\sigma<t_{0} / 3$; we turn to $(2.10)$ of Lemma 2.1 with $j=2$

$$
\begin{aligned}
h_{0}\left(a_{1}, a_{2}\right) & \leq t_{0}+\sigma / 6+a_{2} / 24<t_{0}+\sigma+(1-\sigma) / 48 \\
& \leq t_{0}+\frac{1}{48}+7 \sigma / 48 \leq 1 / 2 .
\end{aligned}
$$

(4.6.2). $a_{1} \geq a_{2}>(2 i+2) t_{0} /(2 i+3)$ and $1-20 t_{0} / 11>\sigma \geq t_{0} / i$.

Proof. If $a_{2} \geq t_{0}$, it is already covered by (4.1.1). If $a_{1} \geq t_{0}>a_{2}>$ $(2 i+2) t_{0} /(2 i+3)$, using (4.1.3). If $t_{0}>a_{1} \geq a_{2}>8 t_{0} / 9$, using (4.2.1).

We can write (4.2.2) to be

(4.6.3). $t_{0} \geq a_{1}>(2 i+2) t_{0} /(2 i+3)$ and $1-20 t_{0} / 11>\sigma \geq t_{0} / i$. (It is not necessary $a_{2} \leq a_{1}$ ).

Proof. If $a_{2} \geq t_{0}$, we are back to (4.1.3). If $a_{1}<t_{0}$, it is covered by (4.2.2).

(4.6.4). $t_{0} \leq a_{1} \leq 1 / 2$, and $\varepsilon<\sigma<1 / 2-8 t_{0} / 9$;

Proof. If $\sigma \geq t_{0} / 5$, then $a_{2}=1-\sigma-a_{1}>8 t_{0} / 9,\left\{\theta_{j}\right\} \in E(\theta)$ by (4.1.3) 
with $i=3$. If $\sigma<t_{0} / 5$, we apply (2.10) with $j=5$ in Lemma 2.1. We have

$$
\begin{aligned}
h_{0}\left(a_{1}, a_{2}\right) & =t_{0} / 2+a_{1} / 2+\sigma / 6+a_{2} / 60 \\
& =t_{0} / 2+a_{1} / 2+\sigma / 6+\left(1-\sigma-a_{1}\right) / 60 \\
& <t_{0} / 2+29 / 120+(9 / 60)\left(1 / 2-8 t_{0} / 9\right)+1 / 60<1 / 2 .
\end{aligned}
$$

(4.6.5). $a_{2} \leq a_{1} \leq 1 / 2$ and $\sigma<1 / 2-8 t_{0} / 9$.

Proof. If $a_{2} \geq t_{0}$, it is covfered by (4.1.1). If $a_{1}<t_{0},\left\{\theta_{j}\right\} \in E(\theta)$ by (4.1.5) since $\sigma<1 / 2-8 t_{0} / 9<1-20 t_{0} / 11$. If $a_{2} \leq t_{0}<a_{1}<1 / 2$, the proof is same as (4.5.6).

From Lemma 4.3 and (4.6.1), we have, for $\sigma<1-20 t_{0} / 11$, (4.4.3) (4.4.8) hold. Moreover, (4.5.3), (4.5.4) and (4.5.5) with $t_{0}<5 / 11$ can imply that

$$
M_{\sigma}-m_{\sigma}>\sigma, \text { if } \sigma<t_{0} / 5
$$

and

$$
M_{\sigma}-m_{\sigma}>t_{0} / 5, \text { if } \sigma \geq t_{0} / 5
$$

since $1-2 t_{0}>t_{0} / 5$.

(4.6.6) $t_{0} \leq a_{1} \leq 1 / 2$ and $a_{2}<2-4 t_{0}$;

In this case, we use Lemma 2.1. By (2.11) with $j=2$ and $a_{1}^{\prime}=a_{1}$,

$$
h_{0}\left(a_{1}, a_{2}\right) \leq a_{1} / 2+t_{0} / 2+a_{2} / 8 \leq 1 / 4+t_{0} / 2+t_{0} / 20<1 / 2 .
$$

Lemma 4.7. Suppose that $t_{0}<5 / 11$ and $L=L_{0}$. If there exists at least one set of complementary partial sums $\left\{a_{1}, a_{2}, \sigma\right\}$ (or $\left\{a_{1}, a_{2}, a_{3}, \sigma\right\}$ ) of $\left\{\theta_{j}\right\}$ such that at least one of conditions (4.7.1) - (4.7.6) holds, then $\left\{\theta_{j}\right\} \in E(\theta)$.

(4.7.1). $k_{0}=3,1 / 2 \geq a_{1} \geq t_{0}, 2 t_{0} / 5 \geq a_{2} \geq t_{0} / 3$, and $t_{0} / 4 \geq a_{3} \geq t_{0} / 5$.

Proof. We use Lemma 3.6 with $a_{2}^{\prime}=\max \left\{a_{2}, t_{0} / 3\right\}=a_{2}$; then

$$
\begin{aligned}
h_{6}\left(a_{1}, a_{2}, a_{3}\right) & =13 t_{0} / 48+\left(a_{1}+a_{2}\right) / 2+5 a_{3} / 16 \\
& \leq 13 t_{0} / 48+1 / 4+t_{0} / 5+5 t_{0} / 64<1 / 2 .
\end{aligned}
$$


(4.7.2). $k_{0}=4, a_{1} \leq 8 t_{0} / 9, a_{2} \leq 4 t_{0} / 9$, and $a_{4} \leq a_{3} \leq t_{0} / 4$.

Proof. Using (3.5), we have that, $a_{3}^{\prime}=a_{4}^{\prime}=t_{0} / 4$ and

$$
h_{4}\left(a_{1}, a_{2}, a_{3}, a_{4}\right)=\frac{29}{64} t_{0}+\max \left\{\frac{3}{40} a_{1}+\frac{a_{2}}{2}+\frac{7 t_{0}}{20}, \frac{a_{1}}{2}+\frac{3 a_{2}}{20}+\frac{t_{0}}{10}\right\}<\frac{1}{2} .
$$

(4.7.3). $k_{0}=4, a_{1}+a_{3}+a_{4} \geq t_{0}, a_{2}+a_{3}+a_{4} \leq t_{0}, a_{1}+a_{3} \leq t_{0}, a_{1}+a_{4} \leq$ $t_{0}, a_{4} \leq a_{3} \leq t_{0} / 5$ and $a_{2} \leq 4-8 t_{0}$.

Proof. Using Lemma 3.7 , since $a_{2} \leq 4-8 t_{0}$, we have

$$
h_{7}\left(a_{1}, a_{2}, a_{3}, a_{4}\right)=t_{0}+a_{2} / 8<1 / 2
$$

(4.7.4). $k_{0}=6,2 t_{0} / 7 \geq a_{1} \geq \cdots \geq a_{0} \geq t_{0} / 5$ and $\sigma+a_{1}+a_{2} \geq t_{0}$.

Proof. Using Lemma 3.8, we have that

$$
h_{8}\left(a_{1}, \cdots, a_{6}\right)=\frac{15}{16} t_{0}+\frac{a_{4}}{12}+\frac{a_{5}}{12}+\frac{19}{48} a_{6} \leq \frac{15}{16} t_{0}+\frac{t_{0}}{21}+\frac{19}{168} t_{0}<\frac{1}{2} .
$$

(4.7.5). $2 t_{0} / 5 \geq \theta_{1} \geq \cdots \geq \theta_{4} \geq 1-20 t_{0} / 11,2 t_{0} / 5 \geq 1-\theta_{1}-\cdots-\theta_{6} \geq \theta_{1}, \geq$ $1-20 t_{0} / 11$, and $\theta_{5} \geq \theta_{B}, 2 \theta_{6} \leq t_{0} / 3, \theta_{1}+\theta_{2}+\theta_{5}<t_{0}<\theta_{1}+\theta_{2}+\theta_{5}+\theta_{B}$.

Proof. In Lemma 3.9, take $a_{1}=\theta_{1}+\theta_{2}, a_{2}=\theta_{3}+\theta_{4}, a_{3}=\theta_{5}$, and $a_{4}=\theta_{6}$, then

$$
\begin{aligned}
a_{4} & =\theta_{6} \leq \frac{1}{2}\left(\theta_{5}+\theta_{6}\right) \leq \frac{1}{2}\left(1-\theta_{2}-\cdots-\theta_{4}-\left(1-\sum_{1 \leq i \leq 8} \theta_{i}\right)\right) \\
& \leq\left(1-5 a_{2}\right) / 2
\end{aligned}
$$

and

$$
\begin{aligned}
h_{9}\left(a_{1}, \cdots, a_{4}\right) & \leq 15 t_{0} / 16+a_{2} / 8+5\left(1-5 a_{2}\right) / 16 \\
& <15 t_{0} / 16+5 / 16-23 a_{2} / 16<1 / 2 .
\end{aligned}
$$

(4.7.6). $k_{0}=4, a_{1}+a_{4} \leq t_{0}, a_{2}=a_{3} \leq t_{0}, \sigma+a_{1} \geq t_{0}, a_{3}<t_{0} / 5, a_{2}<1 / 3$ and $a_{3}<t_{0} / 5$.

Proof. In Lemma 3.10 ,

$$
h_{10}\left(a_{1}, \cdots, a_{4}\right)=t_{0}+a_{2} / 8+a_{3} / 24<t_{0}+1 / 24+t_{0} / 120<1 / 2 .
$$


(4.7.7). $k_{0}=5, a_{3} \leq a_{2} \leq a_{1} \leq t_{0} / 2$, and $a_{5} \leq a_{4} \leq t_{0} / 4$.

Proof. If $a_{2}+a_{1}>8 t_{0} / 9$, we replace $a_{1}$ by $a_{2}+a_{1}$ and $s$ by $a_{5}$ with $k_{0}=2$, then $\left\{\theta_{j}\right\} \in E$ by (4.6.3). If $a_{2}+a_{1} \leq 8 t_{0} / 9$; then $a_{3}>4 t_{0} / 9$ it is covered by (4.7.2).

(4.7.8) $k_{0}=2, a_{1} \geq t_{0} / 2, a_{2} \leq t_{0} / 5$, and $\sigma+a_{2} / 2>t_{0} / 2$.

See Lemma 3.2.

(4.7.9). $k_{0}=2, a_{1} \geq t_{0}, t_{0} / 3 \geq a_{2} \geq t_{0} / 5$, and $\sigma>a_{2} / 8+3 t_{0} / 8$.

See Lemma 3.3.

\section{§ 5. ANALYTIC FORM OF $R\left(x ; M_{1} \cdots M_{k}\right)$.}

We shall examine the remainder term $R\left(x ; M_{1}, \cdots, M_{k}\right)$ (see (1.12)) which was used in [1]. Let $x$ be a large number, $y=x^{\theta}$ with $1 / 2<\theta<7 / 12$, and $\mathbf{A}=\{n: x-y<n<x\}$. For convenience we define $a_{m_{i}, i}=0$, unless $M_{i}<m \leq 2 M_{i}$. We rewrite (1.12):

$$
R\left(x ; M_{1}, \cdots, M_{k}\right)=\sum_{m_{1}, \cdots, m_{k}} a_{m_{1}, 1} \cdots a_{m_{k}, k}\left(\left[\frac{x}{m_{1} \cdots m_{k}}\right]-\left[\frac{x-y}{m_{1} \cdots m_{k}}\right]\right)
$$

and we write

$$
\begin{aligned}
& L=\frac{x}{2 M_{1} \cdots M_{k}}, \\
& L(s)=\sum_{L / 2^{k}<I \leq 3 L} I^{-s}, \\
& M_{i}(s)=\sum_{M_{i}<m_{i} \leq 2 M_{i}}^{L} a_{m_{i}, i} m_{i}^{-*}, \text { for } 1 \leq i \leq k,
\end{aligned}
$$

and

$$
g(s)=L(s) \prod_{1 \leq i \leq k} M_{i}(s)
$$

where $k$ is a positive integer.

Theorem 2. Suppose $\left\{\theta_{j}\right\} \in E(\theta)$, then

$$
R\left(x ; M_{1}, \cdots, M_{k}\right) \ll x^{\theta-\varepsilon} .
$$


Proof. Lemma 3.12 of Titchmarsh [7] is applied to the function $g(3)$, to yield

$$
\sum_{\substack{m_{1}, \cdots, m_{k} \\ I m_{1} \cdots m_{k} \in A}} a_{m_{1}, 1} \cdots a_{n_{k}, k}=\frac{1}{2 \pi i} \int_{c-i T}^{c+i T} g(s) \frac{x^{2}-(x-y)^{\prime}}{s} d s+O\left(\frac{x^{1-\eta}}{T}\right),
$$

where $c=1+(\log x)^{-1}$ and $T>0$. The conditions $I m_{1} \cdots m_{k} \in A$ and $M_{i}<m_{i} \leq 2 M_{i}$ imply that $L / 2^{k}<I \leq 3 L$, and so that the sum on the left. of (5.1) becomes

$$
\begin{aligned}
& \sum_{m_{1}, \cdots, m_{k}} a_{m_{1}, 1} \cdots a_{m_{k}, k}\left(\left[\frac{x}{m_{1} \cdots m_{k}}\right]-\left[\frac{x-y}{m_{1} \cdots m_{k}}\right]\right) \\
& =R\left(x ; M_{1}, \cdots, M_{k}\right)-y \sum_{m_{1}, \cdots, m_{k}} \frac{a_{m_{1}, 1} \cdots a_{m_{k}, k}}{m_{1} \cdots m_{k}}
\end{aligned}
$$

For $T_{0} \leq L$, in the range $\left\{s=c+i t:|t| \leq T_{0} \leq L\right\}$, we have

$$
L(s)=\frac{(3 L)^{1-s}-\left(L / 2^{k}\right)^{1-s}}{s-1}+O\left(L^{-c}\right)
$$

by Theorem 4.11 of Titchmarsh [7]. Moreover if $T_{0} y \leq x$,

$$
\frac{x^{s}-(x-y)^{b}}{s}=y x^{s-1}-O\left(|s| y^{2} x^{c-2}\right) \ll y x^{c-1} \text {. }
$$

Hence

$$
\begin{gathered}
\frac{1}{2 \pi i} \int_{c-i T_{0}}^{c+i T_{0}} g(s) \frac{x^{*}-(x-y)^{*}}{s} d s \\
=\frac{1}{2 \pi i} \int_{c-i T_{0}}^{c+i T_{0}} \frac{x^{\prime}-(x-y)^{s}}{s} M_{1}(s) \cdots M_{k}(s) x^{a-1} d s+O\left(E_{1}\right)+O\left(E_{2}\right),
\end{gathered}
$$

where

$$
E_{1}=\int_{-T_{0}}^{T_{0}} L^{-c}\left|\prod_{1 \leq j \leq k} M_{j}(c-i t)\right| y x^{c-1} d t \ll T_{0} M_{1} \cdots M_{k} y x^{-1},
$$

and

$$
\begin{aligned}
E_{2} & =\int_{-T_{0}}^{T_{0}} L^{-c}\left|\prod_{1 \leq j \leq k} M_{j}(c-i t)\right| y^{2} x^{c-2} d t<T_{0} L^{1-c}\left(M_{1} \cdots M_{k}\right)^{1-c} y^{2} x^{c-2} \\
& \leq T_{0} y^{2} x^{-1}
\end{aligned}
$$


Moreover, on integrating termwise as in the proof of Lemma 3.1 in Titchmarsh [7], we have

$$
\begin{gathered}
\frac{1}{2 \pi i} \int_{c-i T_{b}}^{c+i T_{b}} \frac{x^{-s}-(x-y)^{e}}{s} M_{1}(s) \cdots M_{k}(s) x^{k-1} d s \\
=\sum_{\substack{m_{1}, \cdots, m_{k} \\
1 m_{1} \cdots m_{k} \in A}} \frac{a_{m_{1}, 1} \cdots a_{m_{k}, k}}{m_{1} \cdots m_{k}}+O\left(\frac{1}{T_{0}}\right) .
\end{gathered}
$$

It follows from the above estimate, choosing $T_{0}=L^{1 / 2}$, that

$R\left(x ; M_{1}, \cdots, M_{k}\right)=\frac{1}{2 \pi i}\left(\int_{c-i T}^{c+i T_{0}}+\int_{c+i T_{0}}^{c+i T}\right) g(s) \frac{x^{2}-(x-y)^{\prime}}{s} d s+O\left(x^{\theta-\varepsilon}\right)$.

We divide the latter range into at most $2 \log x$ subintervals of type $\left[T_{1}, T_{2}\right]$, where $T_{2} \leq 2 T_{1}$ and bound the interval over such a range by a sum over well spaced points $T_{1} \leq t_{1}<\cdots<t_{k} \leq T_{2}\left(t_{r+1}-t_{r} \geq 1\right)$. Thus, for some $T_{1}$ with $T_{0} \leq T_{1} \leq T$, and some set of $t_{r}$, we have

$$
R\left(x ; M_{1} \cdots M_{k}\right) \ll x^{\theta-\varepsilon}+(\log x) y x^{c-1} \sum_{1 \leq r \leq k} g\left(c+i t_{r}\right) .
$$

In the following sections we shall estimate (5.3).

We denote the right hand-side of $(5.3)$ by $R\left(x ; N_{1}, \cdots, N_{k_{0}}\right)$. We have

$$
g(s)=N_{1}(s) \cdots N_{k_{0}}(s) L(s),
$$

where the definition of $N_{i}(s)$ or $L_{0}(s)$ is the same as (5.1) and one of $N_{i}(s)$ or $L_{0}(s)$ is equal $L(s) M_{i_{1}}(s) \cdots M_{i_{x}}(s)$.

We trivially have

$$
\left|L_{0}\left(c+i t_{r}\right)\right| \leq 4 \text {, and }\left|N_{j}\left(c+i t_{r}\right)\right| \leq 4 \quad(j \leq k) .
$$

Hence those $t_{r}$ for which

$$
\left|L_{0}\left(c+i t_{r}\right)\right| \leq x^{-2} \text {, or }\left|N_{j}\left(c+i t_{r}\right)\right| \leq x^{-2} \quad\left(j \leq k_{0}\right)
$$

contribute a total

$$
\ll(\log x) y x^{c-1} T_{2} x^{-2} \ll x^{\theta-\varepsilon}
$$


to $R\left(x ; N_{1}, \cdots, l N_{k_{0}}\right)$. Such points may therefore be neglected. We now divide the remaining $t_{r}$ into at mast $(4 \log x)^{k_{0}+1}$ sets $S\left(U_{1}, \cdots, U_{k_{B}}, W\right)$ for which

$$
\begin{aligned}
& U_{i}<N_{i}^{c-1 / 2}\left|N_{i}\left(c+i t_{r}\right)\right|<2 U_{i}, i=1, \cdots, k_{0}, \\
& W \leq L_{0}^{c-1 / 2}\left|L_{0}\left(c+i t_{r}\right)\right|<2 W
\end{aligned}
$$

where $x^{-2} \leq L^{1 / 2-c} W \leq 2^{-u} \leq 1$, for some integer $u$ and similarly for $v_{1}, \cdots, U_{k_{0}}$. It follows that

$R\left(x ; N_{1}, \cdots, N_{k_{0}}\right) \ll x^{6-\varepsilon}+(\log x)^{k_{0}+2} y x^{-1 / 2} U_{1} \cdots U_{k_{0}} W\left|S\left(U_{1}, \cdots, U_{k_{0}}, W\right)\right|$

for some $T$, some $U_{1}, \cdots, U_{k_{0}}, W$ and some well-spaced set $S\left(U_{1}, \cdots, U_{k_{0}}, W\right)$ having the property (1.4) - (1.7): the mean-value technique of Montgomery $[8]$

$$
\left|S\left(U_{1}, \cdots, U_{k_{0}}, W\right)\right| \ll U_{i}^{-2 j}\left(N_{i}^{j}+T\right) \log ^{A} x, 1 \leq i \leq k_{0},
$$

where $A$ is constant; Halasz's method in the form due to Huxley

$$
\left|S\left(U_{1}, \cdots, U_{k_{0}}, W\right)\right| \ll\left(U_{i}^{-2 j} N_{i}+U_{i}^{-B j} N_{i} T\right) \log { }^{A} x
$$

while from the inequality

$$
\left|L_{0}\left(c+i t_{r}\right)\right|^{2} \geq U^{2} L_{0}^{1-2 c},
$$

we have

$$
\left|S\left(U_{1}, \cdots, U_{k_{0}}, W\right)\right| \ll\left(W^{-4} L_{0}^{2}+W^{-12} L_{0}^{2} T\right)(\log x)^{A}
$$

Our last estimate for $\left|S\left(U_{1}, \cdots, U_{k_{0}}, W\right)\right|$ depend on the formula

$$
L(s)=\frac{1}{2 \pi i} \int_{\frac{1}{2}-c-i T}^{\frac{1}{2}-c+i T} \zeta(s+z)\left((3 L)^{z}-\left(\frac{L}{2^{k}}\right)^{z}\right) \frac{d z}{z}+O\left(L^{\frac{1}{2}-c} \log x\right)
$$

for $s=c+i t, T_{1} \leq t \leq 2 T_{1}$. This yield, if $L_{0}=L$,

$$
W^{4}\left|S\left(U_{1}, \cdots, U_{k_{0}}, W\right)\right| \leq L_{0}^{4 c-2} S\left|L_{0}\left(c+i t_{r}\right)\right|^{4} \ll T(\log x)^{A} .
$$


Deshouillers and Iwaniec [6] proved: if $N \geq 1, T \geq 1$, the coefficients $d_{n}$ are complex numbers and $\varepsilon>0$, then

$\int_{-2 T}^{2 T}\left|\zeta\left(\frac{1}{2}+i t\right)\right|^{4}\left|\sum_{n<n \leq 2 N} d_{n} n^{i t}\right|^{2} d t \ll T^{\varepsilon}\left(N_{2} T^{1 / 2}+N^{5 / 4} T^{3 / 4}+T\right) \sum_{n \leq n \leq 2 N}\left|d_{n}\right|^{2}$

From (4.1), we have

$$
\begin{aligned}
& W^{4} U_{j}^{2}\left|S\left(U_{1}, \cdots, U_{k_{0}}, W\right)\right| \leq L^{4 c-2} N^{2 c-1} \sum_{r}\left|L\left(c+i t_{r}\right)\right|^{4}\left|N_{j}\left(c+i t_{r}\right)\right|^{2} \\
& \ll L^{4 c-2} N_{j}^{2 c-1} \sum_{r} \int_{-2 T}^{2 T}\left|\zeta\left(\frac{1}{2}+i t\right)\right|^{4} L^{2-4 c} N_{j}^{1-2 c}\left|N_{j}\left(\frac{1}{2}+i t\right)\right|^{2} \frac{d t}{1+\left|\tau-t_{r}\right|} \\
& \quad+\left|S\left(U_{1}, \cdots, U_{k_{0}}, W\right) \|(\log x)\right|^{4} \ll T^{\varepsilon}\left(N_{k}^{2} T^{1 / 2}+N_{k}^{5 / 4} T^{3 / 4}+T\right)
\end{aligned}
$$

whence, for $1 \leq k \leq j$,

$$
\left|S\left(U_{1}, \cdots, U_{k_{0}}, W\right)\right| \ll W^{-4} U_{k}^{-2}\left(N_{k}^{2} T^{1 / 2}+N_{k}^{5 / 4} T^{3 / 4}+T\right) T^{\varepsilon} .
$$

We have that

$$
U_{1} \cdots U_{k} W\left|S\left(U_{1}, \cdots, U_{k_{0}}, W\right)\right| \ll x^{1 / 2-\varepsilon}+x^{h\left(a_{1}, \cdots, a_{k_{0}}\right)}
$$

with $h\left(a_{1}, \cdots, a_{k_{0}}\right)<1 / 2$ for a certain constant $\varepsilon>0$ since $\left\{\theta_{j}\right\} \in E(\theta)$. This will complete the proof of

$$
R\left(x ; M_{1}, \cdots, M_{k}\right) \ll x^{\theta-\varepsilon} .
$$

Theorem 3. Suppose $\left\{\theta_{j}\right\} \in E(\theta)$. then

$$
\int_{T}^{2 T}\left|W\left(\frac{1}{2}+i t\right)\right| d t \ll x^{\frac{1}{2}-\varepsilon}
$$

for

$$
T_{1} \leq T \leq x^{1-\theta+\varepsilon}
$$

where $\theta$ is a fixed positive constant, and

$$
T_{1}=\exp \left((\log x)^{\frac{1}{3}}(\log \log x)^{-\frac{1}{3}}\right) .
$$


Proof. Defined $S\left(U_{1}, \cdots, U_{k}, W\right)$ be a set of $t_{r}$ into for which

$$
\begin{aligned}
& U_{i}<M_{i}^{c-1 / 2}\left|M_{i}\left(c+i t_{r}\right)\right|<2 U_{i,} i=1, \cdots, k_{0}, \\
& W \leq L_{0}^{c-1 / 2}\left|L_{0}\left(c+i t_{r}\right)\right|<2 W,
\end{aligned}
$$

where $x^{-2} \leq L^{1 / 2-c} W \leq 2^{-u} \leq 1$, for some integer $u$ and similarly for $U_{1}, \cdots, U_{k_{0}}$. In Lemma 16 of [8], Heath-Brown proved

$$
\int_{T}^{2 T}\left|W\left(\frac{1}{2}+i t\right)\right| d t<x^{\frac{1}{2}-\varepsilon}+x^{-\delta} \sum\left|S\left(U_{1}, \cdots, U_{k}, W\right)\right|
$$

where $\sum$ runs over $\left(U_{1}, \cdots, U_{k}, W\right)$ such that (1.1), (1.2) and (1.3), and $\left|S\left(U_{1}, \cdots, U_{k}, W\right)\right|$ satisfies (1.4), (1.5), (1.6), (1.7) and (1.9). Since $\left\{\theta_{j}\right\} \in$ $E(\theta)$, we have that

$$
\left|S\left(U_{1}, \cdots, U_{k}, W\right)\right|<x^{\frac{1}{2}-\varepsilon}
$$

then

$$
\left.\sum \mid S\left(U_{1}, \cdots, U_{k}, W\right)\right) \mid<x^{\frac{1}{2}-\epsilon}
$$

since at most $(4 \log x)^{k+1}$ terms in above " $\sum$ ". Thus (5.14) follows. 


\section{References}

[1] Heath-Brown, D.R. and Iwaniec, H., On the Difference Between Consecutive Primes, Invent. Math. 55, 49-69 (1979).

[2] Heath-Brown, D.R., The Number of Primes in a Short Interval, J. Reine Angew, Math. 389, 22-63 (1988).

[3] Lou, S. and Yao, Q., The Number of Primes in a Short Interval, HardyRamanujan J., 16 (1993), 21-43.

[i] Lou, S. and Yao, Q., A Chebychev's Type of Prime Number Theorem in a Short Interval-II, Hardy-Ramanujan J., 15 (1992), 1-33.

[5] Lou, S. and Yao, Q., On the Difference Between Consecutive Primes, (to be submitted).

[6] Deshouillers J.-M. and Iwaniec H., Power mean-value of the Riemann zeta-function, Mathematika, 29 (1982), 202-212.

[7] Titchmarsh E.C., The Theory of the Riemann zeta-function, Oxford (1951).

[8] Montgomery H.L., Topics in Multiplicative Number Theory, Lecture Notes in Mathematics, Springer-Verlag, 227 (1971).

Lou, Shituo and Yao, Qi

Department of Math.

Dalhousie University

Halifax, Nova Scotia B3H 3J5

Canada 